Math. Model. Nat. Phenom.

Vol. 7, No. 6, 2012, pp. 167-186

DOI: $10.1051 / \mathrm{mmnp} / 20127608$

\title{
Membrane Associated Complexes: New Approach to Calcium Dynamics Modelling
}

\author{
M. Dyzma ${ }^{1}$, P. Szopa ${ }^{1,2}$, B. Kaźmierczak ${ }^{1}$ * \\ ${ }^{1}$ Institute of Fundamental Technological Research, Polish Academy of Sciences, Warsaw \\ ${ }^{2}$ Faculty of Mathematics, Informatics and Mechanics, University of Warsaw
}

\begin{abstract}
Mitochondria are one of the most important organelles determining $\mathrm{Ca}^{2+}$ regulatory pathway in the cell. Recent experiments suggested the existence of cytosolic microdomains with locally elevated calcium concentration (CMDs) in the nearest vicinity of the outer mitochondrial membrane (OMM). These intermediate physical connections between endoplasmic reticulum (ER) and mitochodria are called MAM (mitochondria-associated ER membrane) complexes.

The aim of this paper is to take into account the direct calcium flow from ER to mitochondria implied by the existence of MAMs and perform detailed numerical analysis of the influence of this flow on the type and shape of calcium oscillations. Depending on the permeability of MAMs interface and ER channels, different patterns of oscillations appear (simple, bursting and chaotic). For some parameters the oscillatory pattern disappear and the system tends to a steady state with extremely high calcium level in mitochondria, which can be interpreted as a crucial point at the beginning of an apoptotic pathway.
\end{abstract}

Keywords and phrases: $\mathrm{Ca}^{2+}$ signaling, mitochodrial $\mathrm{Ca}^{2+}$ transport, MAMs, three pool model, bistability, apoptosis,

Mathematics Subject Classification: 34A34, 34B60, 34C60

\section{Introduction}

Cells use much of their energy to cause spatial and temporal gradients in $\mathrm{Ca}^{2+}$ concentration and to maintain over ten thousand fold differences between their cytosolic $(0.1-1 \mu \mathrm{M})$ and extracellular $(2$ $\mathrm{mM}$ ) concentrations [1]. $\mathrm{Ca}^{2+}$ concentration differs also between specific cell organelles: mitochondria, endoplasmic reticulum (ER) and nucleus. In these compartments it ranges between $0.2-500 \mu \mathrm{M}, 100-$ $500 \mu \mathrm{M}$ and $0.1-2 \mu \mathrm{M}$, respectively [2].

Calcium homeostasis is controlled by interactions of pumps, channels and exchangers operating inside the cell. Sarco-endoplasmic reticulum $\mathrm{Ca}^{2+}$ ATPase (SERCA) pumps calcium from the cytosol to the lumen of $\mathrm{ER}$, while $\mathrm{IP}_{3} \mathrm{R}$ or ryanodine receptors ( $\mathrm{RyR}$ ) release calcium from the ER stores. Voltagedependent anion channels (VDAC) on outer mitochondrial membrane and uniporters on the internal mitochondrial membrane (IMM) allow $\mathrm{Ca}^{2+}$ to reach mitochondrial matrix along the electro-chemical gradient [3], [4]. $\mathrm{Ca}^{2+}$ can exit mitochondria through specific exchangers located on the internal mitochondrial membrane, which remove calcium from the matrix to the perimitochondrial space, which

*Corresponding author. E-mail: bkazmier@ippt.gov.pl 
separates the inner and outer mitochondrial membranes, and further to the cytosol, replacing it with sodium or hydrogen ions, i.e. mitochondrial sodium-calcium exchanger (MNCX) [5]. In particular conditions, when mitochondria uptake vast amount of calcium, it may be released by specific mitochondrial permeability transition pores (PTPs). Opening of PTPs, according to [6] and [7], is considered to be a crucial control point at the apoptotic pathway [8]. Most of the cells show simple calcium oscillations. Sometimes more complex periodic behavior is noted, for example bursting oscillations of pancreatic acinar cells treated with carbahol or in endothelial cells activated by ATP [9].

\section{MAM microdomains}

First reports concerning existence of physical interface between ER and mitochondria were announced by Dennis and Kennedy who described so called "fraction x" isolated from rat hepatocytes and tested for mitochondrial and reticular enzymes [12]. This "fraction $\mathrm{x}$ " was identified 20 years later as mitochondria and endoplasmic reticulum complexes involved in lipids' metabolism [13]. Recent experiments demonstrated that mitochondria and ER indeed form functional complexes with each other [14]. These complexes are known as the mitochondria-associated ER membrane complexes - MAMs [15]. A scheme and a picture of MAM is presented in Figs. 1 and 3.

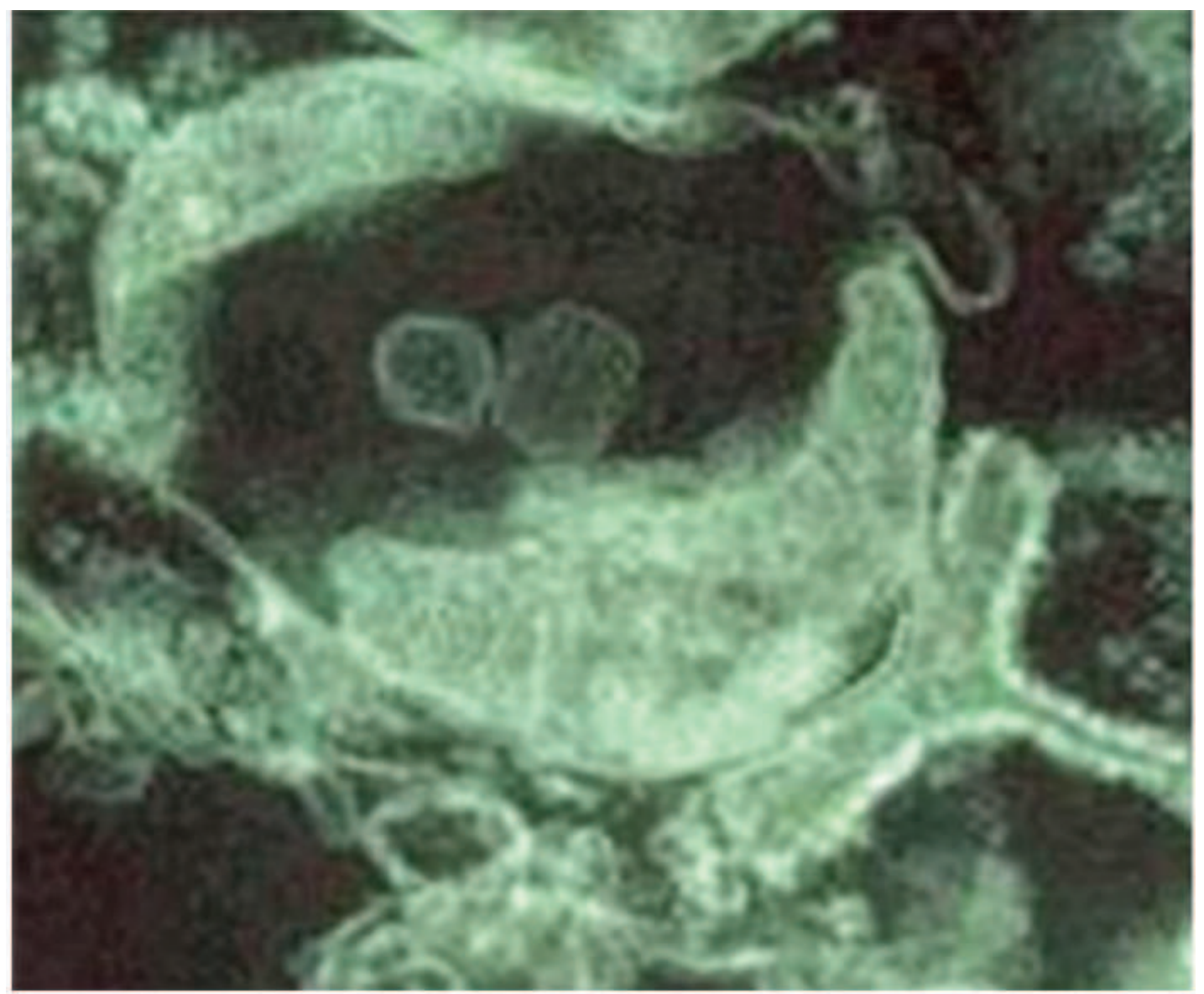

FiguRE 1. Mitochondria-associated ER membrane complex (MAMs) - microscopic picture (by Mariusz R. Wieckowski) 
The distance between membranes in MAMs ranges from $10 \mathrm{~nm}$ for smooth ER to $25 \mathrm{~nm}$ for rough ER [17]. Appositions of ER and OMM are stabilized by several proteins e.g. VDAC-1 channels and $\mathrm{IP}_{3} \mathrm{R}$ type-3/RyR, forming ER-mitochondrion interface [18].

The results of Csordas and Giacomello ([11], [16]) indicate that during stimulations, calcium concentration in microdomains is 5 to 10 -fold higher than in the bulk cytosol and may reach up to $9 \mu \mathrm{M}$. Therefore in our opinion it is reasonable to use reticular calcium concentration in equation describing current $J_{M A M}$ (see section 3, equation (3.17)).

\section{The mathematical model}

The models proposed in references [10], [19] and [20] describe the evolution of calcium in three specified cellular compartments: endoplasmic reticulum, mitochondria and cytosol. The cytosolic calcium level depends on fluxes across ER membrane, mitochondria membrane and binding of free $\mathrm{Ca}^{2+}$ to cytosolic buffer proteins ([22], [23], [24]).

In the above models, the exchange of calcium between the endoplasmic reticulum and the mitochondria is only via cytosol. However, in the papers by Marhl and collaborators ([10], [21]) the existence of MAMs was suggested by taking relatively low half-activation constant of the uniporter.

In the model presented in this paper we take into account the sites of direct contact between ER and mitochodrion - MAMs which imply the direct calcium flow from ER to mitochondrion. The model is based on the paper [10] and its scheme is presented in Fig. 2.

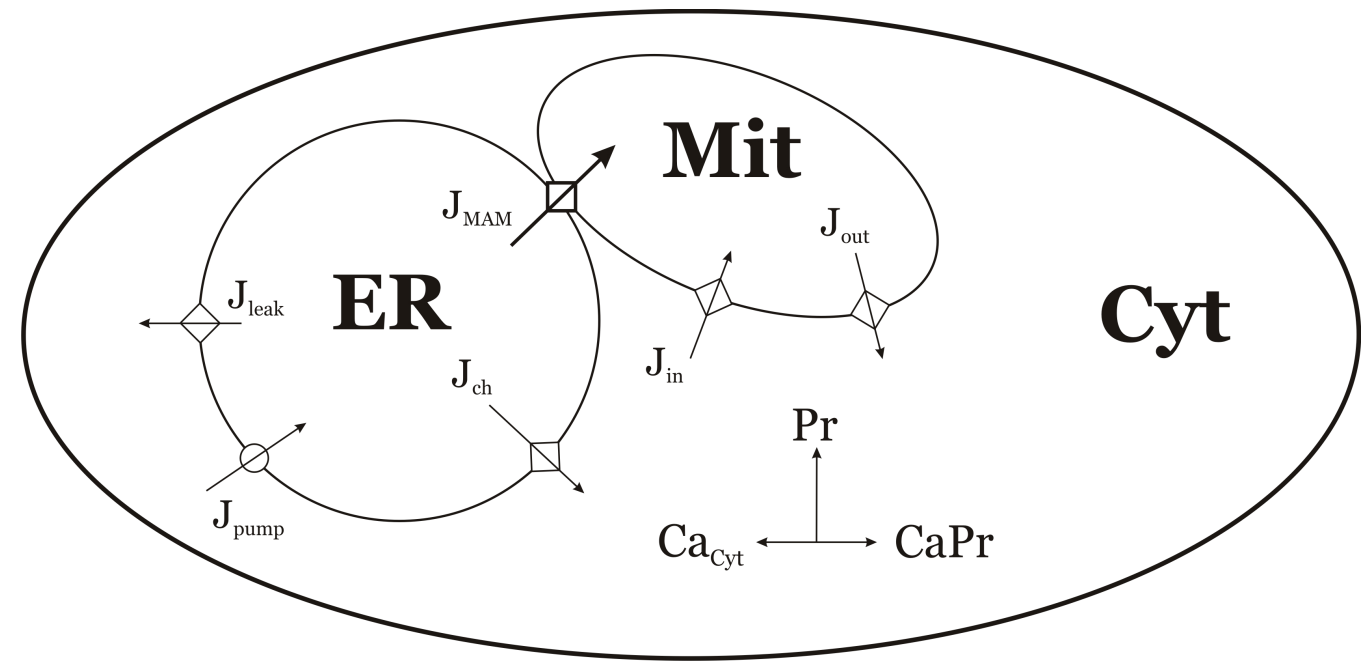

FiguRE 2. Schematic representation of the model: ER - endoplasmic reticulum, Mit mitochondrion, Cyt - cytosol

It is given by the system (3.1)-(3.5). From the mathematical point of view, the main difference with the model in [10] is an additional flux $J_{M A M}$ defined by (3.17), describing the flow of free calcium straightforwardly from ER to mitochondria in microdomains corresponding to MAM-regions. The intensity of the flux depends on the value of the calcium concentration in the close vicinity of endoplasmic reticulum $\mathrm{Ca}_{E R}$. (See also the remarks in the last paragraph of this section.) As we mentioned above in [21], the existence of sites of close proximity of ER and mitochondria (MAMs) was modelled by taking relatively small half-activation constant of the uniporter $K_{2}$. Obviously these two approaches are not equivalent from the mathematical point of view. Moreover in our approach we have obtained biologically reasonable 
scenario of calcium induced apoptosis. That is for large values of $k_{M A M}$ parameter we observe that mitochondria accumulate large amount of calcium ions, this process is called 'mitochondria swelling', which is an early step in calcium induced apoptotic pathway (see section Biologically acceptable apoptosis scenario).

We use the system of ODEs to describe the evolution of calcium concentrations in different cellular compartments. Although such a description is not strictly legitimate, this can be partially justified by considering the diffusion time of calcium inside the cytosol. The diffusion coefficient $D_{c}$ of free calcium is about $300 \mu \mathrm{m}^{2} / \mathrm{s}$ [25], whereas the diffusion coefficients of most of the buffering particles is several times smaller and sometimes may be even regarded as zero. (However, for some buffer particles it may be comparable to the diffusion coefficient of free calcium.) The effective diffusion coefficient of calcium $D_{\text {eff }}$ in the presence of buffers depends on the kind of cells considered, but it is usually assumed that [26]

$$
D_{\text {eff }}=20 \mu \mathrm{m}^{2} / \mathrm{s} .
$$

Therefore, the mean distance covered by calcium ions in time $t$ is equal to $\sqrt{D_{\text {eff }} \times t}$. Thus, according to the above estimates, time necessary to equalise the calcium concentration in the cell of diameter close to typical one (about $6 \mu \mathrm{m}$ ) can be estimated as $1.8 \mathrm{~s}$. It is thus much smaller (though comparable) than the characteristic bursting calcium oscillations periods in this paper, which are of the order of $15 \mathrm{~s}$ and the period of simple oscillations depicted in Figs. 4 is about 11 seconds. So, despite the fact that the calcium channels form clusters distributed non-homogeneously on the membranes of ER [27] and mitochondria [28], [29] and the fact that the mitochondria themselves may be gathered in the parts of the cell which require more intensive energy supply, we can neglect the non-homogeneities of calcium stores distribution inside the cell and use ODEs to describe the evolution of spatially averaged concentration of calcium in different compartments if the size of the cell is not too large (i.e. does not exceed much $6 \mu \mathrm{m}$ ).

Another problem in applying spatially homogeneous models described by ODEs is a stochastic nature of a single calcium channel activity which takes the form of irregular in time opening events called 'blips'. This can further lead to a stochastic activation of other channels within the same cluster (called 'puffs') [27]. However, in cells with large population of channels bursting may occur in a quasi-synchronized manner in response to sufficiently strong external stimulus, thus it may be justified to use spatially homogeneous models. Such a situation takes place even in relatively large cells such as hepatocytes [30].

The concentrations of free calcium ions in the cytosol $\left(\mathrm{Ca}_{C y t}\right)$, endoplasmic reticulum $\left(\mathrm{Ca}_{E R}\right)$ and mitochondria $\left(\mathrm{Ca}_{\mathrm{Mit}}\right)$ as well as the concentration of calcium ions bound to buffer proteins in the cytosol $(\mathrm{CaPr})$ and the concentration of free binding sites of the cytosolic buffer proteins (Pr) are subject to the following equations:

$$
\begin{aligned}
\frac{d \mathrm{Ca}_{C y t}}{d t} & =J_{\text {ch }}+J_{\text {leak }}-J_{\text {pump }}+J_{\text {out }}-J_{\text {in }}+k_{-} \mathrm{CaPr}-k_{+} \mathrm{Ca}_{C y t} \mathrm{Pr} \\
\frac{d \mathrm{Ca}_{E R}}{d t} & =\frac{\beta_{E R}}{\rho_{E R}}\left(J_{\text {pump }}-J_{\text {ch }}-J_{\text {leak }}-J_{M A M}\right) \\
\frac{d \mathrm{Ca}_{M i t}}{d t} & =\frac{\beta_{M i t}}{\rho_{M i t}}\left(J_{\text {in }}+J_{M A M}-J_{\text {out }}\right) \\
\frac{d \mathrm{CaPr}}{d t} & =k_{+} \mathrm{Ca}_{C y t} \mathrm{Pr}-k_{-} \mathrm{CaPr} \\
\frac{d \mathrm{Pr}}{d t} & =-k_{+} \mathrm{Ca}_{C y t} \mathrm{Pr}+k_{-} \mathrm{CaPr}
\end{aligned}
$$

where $k_{+}$and $k_{-}$denote average kinetic constants of $\mathrm{Ca}^{2+}$ binding to the buffer proteins and the reverse process, whereas $\rho_{E R}$ and $\rho_{M i t}$ denote the volume ratio between the ER and the cytosol, and between the mitochondria and the cytosol. We assume that calcium sequestration in ER and mitochondria by buffers is very fast, much faster than the fluxes to and from these cellular compartments ([31], [32], [33], [34]), therefore for simplicity we use the quasi steady state approximation, i.e. we assume (as in [10]) that the ratio of the concentrations of free calcium in the ER and mitochondria to the respective total 
concentration of calcium (free and buffered) is constant and equal to $\beta_{E R}$ and $\beta_{M i t}$, respectively. We have implicitly assumed that there is no calcium exchange between the cell and the extracellular space. Thus the following conservation relation for the total amount of calcium holds

$$
\mathrm{Ca}_{t o t}=\mathrm{Ca}_{C y t}+\frac{\rho_{E R}}{\beta_{E R}} \mathrm{Ca}_{E R}+\frac{\rho_{M i t}}{\beta_{M i t}} \mathrm{Ca}_{M i t}+\mathrm{CaPr} .
$$

This assumption can be justified because usually the calcium transport across the cell membrane is much slower than the calcium transport between the internal compartments [35]. Additionally, according to [35], calcium oscillations can still occur, provided that the total amount of $\mathrm{Ca}^{2+}$ in the cell is in a proper range, even if calcium transport across the plasma membrane is blocked. Adding equations (3.4) and (3.5) we obtain $\frac{d}{d t}(\mathrm{CaPr}+\mathrm{Pr})=0$, which implies that the total concentration $\operatorname{Pr}_{\text {tot }}$ of calcium binding sites (i.e. those with calcium ions bound and those with no calcium ions bound) is constant, that is to say:

$$
\operatorname{Pr}(t)+\operatorname{CaPr}(t)=\operatorname{Pr}_{\text {tot }}(t)=\operatorname{Pr}_{\text {tot }}(0)=\operatorname{Pr}(0)+\operatorname{CaPr}(0) .
$$

By means of conservation relations (3.6) and (3.7) the system of equations (3.1)-(3.5) may be reduced to a three-dimensional one

$$
\begin{aligned}
\frac{d \mathrm{Ca}_{C y t}}{d t} & =J_{\text {ch }}+J_{\text {leak }}-J_{\text {pump }}+J_{\text {out }}-J_{\text {in }}+k_{-} \mathrm{CaPr}-k_{+} \mathrm{Ca}_{C y t}\left(\operatorname{Pr}_{\text {tot }}-\mathrm{CaPr}\right), \\
\frac{d \mathrm{Ca}_{E R}}{d t} & =\frac{\beta_{E R}}{\rho_{E R}}\left(J_{\text {pump }}-J_{\text {ch }}-J_{\text {leak }}-J_{M A M}\right), \\
\frac{d \mathrm{Ca}_{M i t}}{d t} & =\frac{\beta_{\text {Mit }}}{\rho_{\text {Mit }}}\left(J_{\text {in }}+J_{\text {MAM }}-J_{\text {out }}\right),
\end{aligned}
$$

where

$$
\mathrm{CaPr}=\mathrm{Ca}_{t o t}-\mathrm{Ca}_{C y t}-\frac{\rho_{E R}}{\beta_{E R}} \mathrm{Ca}_{E R}-\frac{\rho_{M i t}}{\beta_{M i t}} \mathrm{Ca}_{M i t} .
$$

The active influx of $\mathrm{Ca}^{2+}$ into ER lumen $J_{\text {pump }}$ performed by SERCA pumps and the effluxes $J_{c h}$ and $J_{\text {leak }}$ from this compartment are given by:

$$
\begin{aligned}
J_{\text {pump }} & =k_{\text {pump }} \mathrm{Ca}_{C y t}, \\
J_{c h} & =k_{c h} \frac{\mathrm{Ca}_{C y t}^{2}}{K_{1}^{2}+\mathrm{Ca}_{C y t}^{2}}\left(\mathrm{Ca}_{E R}-\mathrm{Ca}_{C y t}\right), \\
J_{\text {leak }} & =k_{\text {leak }}\left(\mathrm{Ca}_{E R}-\mathrm{Ca}_{C y t}\right) .
\end{aligned}
$$

$J_{\text {pump }}$ depends directly on cytoplasmic $\mathrm{Ca}^{2+}$ concentration with the $k_{\text {pump }}$ as a rate constant of the pump. Calcium ions may exit ER lumen via $\mathrm{IP}_{3} \mathrm{R} / \mathrm{RyR}$ channels $\left(J_{c h}\right)$. This flow depends on $\left(\mathrm{Ca}_{E R}{ }^{\left.-C \mathrm{Ca}_{C y t}\right) ; k_{c h}}\right.$ denotes the maximal permeability of the channels. The Hill function in the expression for $J_{c h}$ represents the CICR (calcium-induced calcium release) mechanism and $K_{1}$ is the half-saturation constant. We also consider non-specific leak flux $J_{\text {leak }}$ which driving force is the concentration difference across the ER membrane; $k_{\text {leak }}$ denotes rate constant for $\mathrm{Ca}^{2+}$ leakage through the membrane of the ER.

The mitochondrial calcium dynamics is determined by the following fluxes:

$$
\begin{aligned}
J_{\text {out }} & =\left(k_{\text {out }} \frac{\mathrm{Ca}_{C y t}^{2}}{K_{3}^{2}+\mathrm{Ca}_{C y t}^{2}}+k_{m}\right) \mathrm{Ca}_{M i t}, \\
J_{\text {in }} & =k_{\text {in }} \frac{\mathrm{Ca}_{C y t}^{8}}{K_{2}^{8}+\mathrm{Ca}_{C y t}^{8}}, \\
J_{M A M} & =k_{M A M} \frac{\mathrm{Ca}_{E R}^{8}}{K_{4}^{8}+\mathrm{Ca}_{E R}^{8}} .
\end{aligned}
$$


The slow calcium release from mitochondria takes place through $\mathrm{Na}^{+} / \mathrm{Ca}^{2+}$ and $\mathrm{H}^{+} / \mathrm{Ca}^{2+}$ exchangers. This flow has only minimal influence on the transmembrane potential $\Delta \Psi_{m}$ ([36], Sec. 3.4 in [37]), thus the changes in $\Delta \Psi_{m}$ are neglected in the model formulation. Taking also into account a very small non-specific leak $k_{m} \mathrm{Ca}_{M i t}$, we express the calcium efflux from mitochondria $J_{\text {out }}$ as given above. $k_{m}$ is the rate constant of the non-specific leak flux, $k_{\text {out }}$ stands for the maximal rate of calcium flow through $\mathrm{Na}^{+} / \mathrm{Ca}^{2+}$ and $\mathrm{H}^{+} / \mathrm{Ca}^{2+}$ exchangers and $K_{3}$ is the half-saturation constant. $J_{i n}$ describes transport of free calcium ions from cytosol into the mitochondria through a specific uniporter (cf. [39]), driven by the electrochemical gradient across the mitochondrial membrane $\Delta \Psi_{m}$. The uniporter is very fast and effective at outer calcium level of more than about $0.5 \mu \mathrm{M}$ (cf. [37], [40]) and much less effective for smaller concentrations. We model this step like kinetics, by taking the Hill coefficients for $J_{i n}$ and $J_{M A M}$ equal to 8 , as in [10]. The constants $k_{i n}$ and $K_{2}$ denote the maximal uniporter permeability and halfsaturation constant for free cytosolic calcium. The value of a transmembrane potential $\Delta \Psi_{m}$ which gives rise to this flow is implicitly taken into account in $k_{i n}$. In our model we will assume that $\Delta \Psi_{m}=$ const. The only factor able to change transmembrane potential $\Delta \Psi_{m}$ is the massive efflux of the matrix contents (in particular $\mathrm{Ca}^{2+}$ ions) through PTPs. Though some papers consider the outflow of calcium through PTPs (in low conductance state) [4], [10], it seems that PTPs opening takes place extremely rarely and mainly under stress conditions e.g. in the process of apoptosis [38]. Thus the possible flow through PTPs is not taken into account. As a result, in the expression for $J_{i n}$ there is no term corresponding to the difference of calcium concentration inside and outside the mitochondria ([41]).

As mentioned above, the main objective of this paper is to incorporate into a mathematical model the existence of regions of very close proximity of endoplasmic reticulum and mitochondria implying the possibility of straightforward flux of free calcium ions from ER to mitochondria. It has been proved recently ([16], [11]), that upon activation calcium concentration in these structures rises rapidly (up to 9 $\mu \mathrm{M})$, so is much higher than in the bulk cytosol.
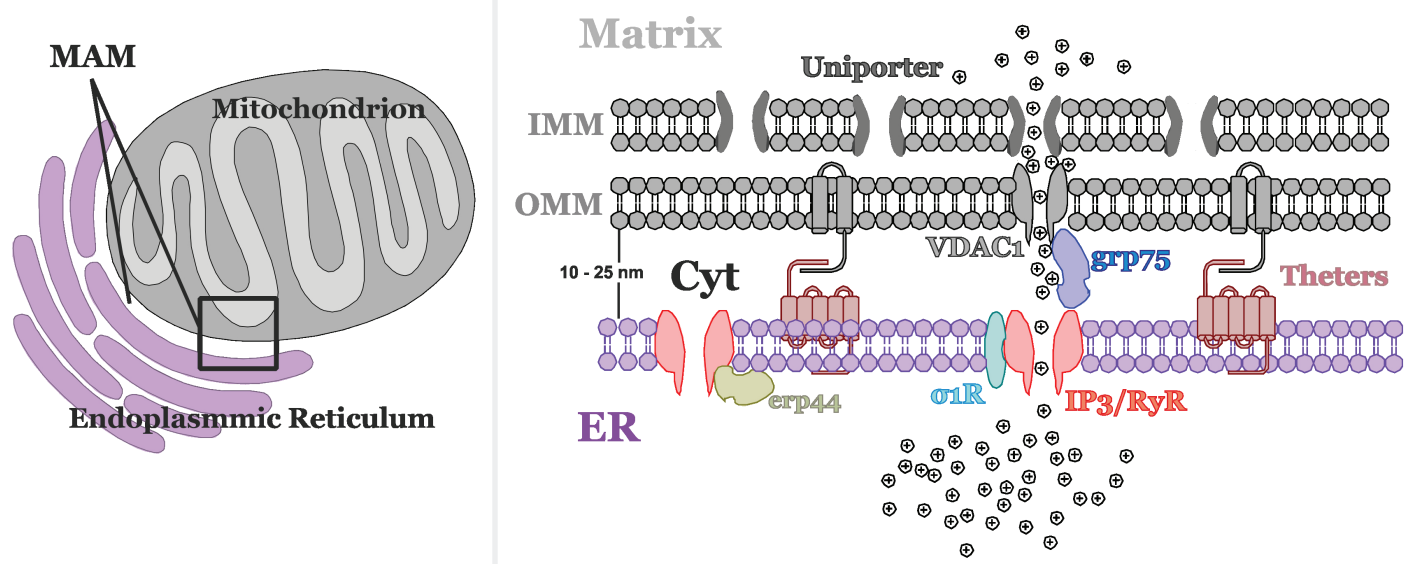

Mitochondria associated endoplasmic reticulum complex

Figure 3. Mitochondria-associated ER membrane complex (MAM), ER - endoplasmic reticulum, Cyt - cytosol, $\mathrm{IP}_{3} \mathrm{R} / \mathrm{RyR}$ - calcium channels on the surface of the ER, VDAC-1/uniporter - calcium channels on outer mitochondria membrane, erp44/erp57 endoplasmic reticulum protein $44 / 57, \sigma_{1} \mathrm{R}$ - sigma-1 receptor and grp75 chaperone 
Additionally, according to [5], majority of the specific uniporters distributed on the inner mitochondrial membrane are located in the regions of close proximity of the endoplasmic reticulum and the outer mitochondrial membrane (see Fig. 1), thus creating mitochondrial associated ER membrane complexes (MAMs). Due to the proximity of ER and OMM in microdomains, calcium released from ER quickly reaches local equilibrium equal to lumenal concentration. Therefore calcium influx through the uniporters located at the inner mitochondrial membrane can be regarded as a straightforward flow from ER to mitochondria. This flux, denoted by $J_{M A M}$, takes place along the concentration gradient. The constants $k_{M A M}$ and $K_{4}$ denote the maximal permeability of the MAMs and half-saturation constant for free reticular calcium. Since the calcium is taken up by mitochondria through the same uniporters regardless of the presence of ER in the neighbourhood of OMM, we postulate $J_{M A M}$ to have the same form as $J_{i n}$ i.e. the same Hill coefficient. However, due to the close proximity of uniporters to endoplasmic reticulum, we will assume below that the permeability of the MAM's uniporters depends straightforwardly on the free calcium concentration in endoplasmic reticulum $\mathrm{Ca}_{E R}$ (see [5], p. 72, [16]). This is the main quantitative difference between $J_{i n}$ and $J_{M A M}$. The reference values of all but $k_{M A M}$ and $K_{4}$ parameters used throughout this paper are the same as in [10]. For the reference values of $k_{M A M}$ and $K_{4}$, given in Table 1 , the majority $(\approx 80 \%)$ of calcium released from ER is sequestered in mitochondria (see section 4 for details). Moreover, this property is robust, i.e. remains valid in the wide range around these values.

For all simulations, unless stated otherwise, we have used the values of parameters given in Table 1. In the case we have changed the parameters, we provide only the values of changed ones.

\begin{tabular}{lll|lll}
\hline Parameter & \multicolumn{2}{c}{ Value } & Parameter & \multicolumn{2}{c}{ Value } \\
\hline Concentrations & & & Kinetic parameters & & \\
Catot & 90 & $\mu \mathrm{M}$ & $k_{\text {ch }}$ & 4100 & $\mathrm{~s}^{-1}$ \\
Pr $_{\text {tot }}$ & 120 & $\mu \mathrm{M}$ & $k_{\text {pump }}$ & 20 & $\mathrm{~s}^{-1}$ \\
Ratios of volumes & & & $k_{\text {leak }}$ & 0.05 & $\mathrm{~s}^{-1}$ \\
$\rho_{\text {ER }}$ & \multirow{2}{*}{0.01} & & $k_{\text {in }}$ & 300 & $\mu \mathrm{Ms}^{-1}$ \\
$\rho_{\text {Mit }}$ & 0.01 & & $k_{\text {out }}$ & 125 & $\mathrm{~s}^{-1}$ \\
& & & $k_{m}$ & 1200 & $\mu \mathrm{Ms}^{-1}$ \\
Ratios of concentrations & & & $k_{+}$ & 0.00625 & $\mathrm{~s}^{-1}$ \\
$\beta_{\text {ER }}$ & 0.0025 & $k_{-}$ & 0.1 & $\mu \mathrm{M}^{-1} \mathrm{~s}^{-1}$ \\
$\beta_{\text {Mit }}$ & 0.0025 & $K_{1}$ & 0.01 & $s^{-1}$ \\
& & & $K_{2}$ & 5 & $\mu \mathrm{M}$ \\
& & $K_{3}$ & 0.8 & $\mu \mathrm{M}$ \\
& & & $K_{4}$ & 5 & $\mu \mathrm{M}$ \\
\hline
\end{tabular}

TABLE 1. The parameters of the model

\section{Results}

First, we show basic mathematical properties of the model, that is the existence and uniqueness of nonnegative global solutions. Next, we perform the numerical analysis. We examine the existence and stability of steady states, existence, period and type (simple, bursting or chaotic) of calcium oscillations. As bursting oscillations we understand the oscillations which are characterized by the superposition of the low amplitude high frequency and high amplitude low frequency oscillations (cf. upper panels of Fig. 5).

The main field of our interest in this paper are differences between the Marhl model [10] and our model with an additional direct calcium flux from ER to mitochondria. 


\subsection{Existence and non-negativity of solutions}

First we show that for non-negative initial conditions the solutions remain non-negative. This property is important from biological point of view, because concentrations can not become negative. To show the non-negativity of solutions we will use the following theorem from [42].

Theorem 4.1. (Proposition 1.1 in [42]) The cone $\mathbb{R}_{+}^{N}$ is invariant for the flow generated by equation

$$
\frac{d u}{d t}=F(u)
$$

if an only if the function $F(u)$ is quasi positive, i.e. for every $i=1, \ldots, N$ the function

$$
F_{i}\left(u_{1}, \ldots, 0, \ldots, u_{N}\right) \geq 0
$$

where 0 stands at $i$-th position and $u_{j} \geq 0$ for $j \neq i$.

Here we use the system of equations in the original form (3.1)-(3.5).

Theorem 4.2. Assume that $\mathrm{Ca}_{C y t}(0), \mathrm{Ca}_{E R}(0), \mathrm{Ca}_{M i t}(0), \mathrm{CaPr}(0)$ and $\operatorname{Pr}(0)$ are non-negative. Then the solutions to equations (3.1)-(3.5) are unique, global in time and non-negative.

Proof. First, using Theorem 4.1, we prove the non-negativity of solutions. Then we prove the boundedness and thus the global existence.

In our case $N=5$ and

$$
\begin{aligned}
F_{1}\left(0, \mathrm{Ca}_{E R}, \mathrm{Ca}_{M i t}, \mathrm{CaPr}, \mathrm{Pr}\right) & =k_{\text {leak }} \mathrm{Ca}_{E R}+k_{m} \mathrm{Ca}_{M i t}+k_{-} \mathrm{CaPr}, \\
F_{2}\left(\mathrm{Ca}_{C y t}, 0, \mathrm{Ca}_{M i t}, \mathrm{CaPr}, \mathrm{Pr}\right) & =\frac{\beta_{E R}}{\rho_{E R}}\left(k_{p u m p} \mathrm{Ca}_{C y t}+k_{c h} \frac{\mathrm{Ca}_{C y t}^{3}}{K_{1}^{2}+\mathrm{Ca}_{C y t}^{2}}+k_{\text {leak }} \mathrm{Ca}_{C y t}\right), \\
F_{3}\left(\mathrm{Ca}_{C y t}, \mathrm{Ca}_{E R}, 0, \mathrm{CaPr}, \mathrm{Pr}\right) & =\frac{\beta_{M i t}}{\rho_{M i t}}\left(k_{i n} \frac{\mathrm{Ca}_{C y t}^{8}}{K_{2}^{8}+\mathrm{Ca}_{C y t}^{8}}+k_{M A M} \frac{\mathrm{Ca}_{E R}^{8}}{K_{4}^{8}+\mathrm{Ca}_{E R}^{8}}\right), \\
F_{4}\left(\mathrm{Ca}_{C y t}, \mathrm{Ca}_{E R}, \mathrm{Ca}_{M i t}, 0, \mathrm{Pr}\right) & =k_{+} \mathrm{Ca}_{C y t} \mathrm{Pr}, \\
F_{5}\left(\mathrm{Ca}_{C y t}, \mathrm{Ca}_{E R}, \mathrm{Ca}_{M i t}, \mathrm{CaPr}, 0\right) & =k_{-} \mathrm{CaPr} .
\end{aligned}
$$

The above functions are obviously non-negative for non-negative $\mathrm{Ca}_{C y t}, \mathrm{Ca}_{M i t}, \mathrm{Ca}_{E R}, \mathrm{CaPr}$ and $\mathrm{Pr}$, thus the solutions are non-negative. We consider a cell as an isolated system i.e. there are no fluxes between the cell volume and the extracellular space. It follows from the conservation of total $\mathrm{Ca}^{2+}$ amount (3.6) and non-negativity of solutions that $\mathrm{Ca}_{C y t}(t), \frac{\rho_{E R}}{\beta_{E R}} \mathrm{Ca}_{E R}(t)$ and $\frac{\rho_{M i t}}{\beta_{M i t}} \mathrm{Ca}_{M i t}(t)$ are bounded above by $\mathrm{Ca}_{t o t}$, while $\operatorname{Pr}(t)$ and $\operatorname{CaPr}(t)$ are bounded above by $\operatorname{Pr}_{t o t}$. The functions $F_{1}-F_{5}$ are polynomials or rational functions with denominators separated from 0 , thus satisfying the local Lipschitz condition. It follows that the solutions to system (3.1)-(3.5) are global in time and unique.

\subsection{Numerical analysis}

The main areas of our investigation here are: 1) influence of MAMs on the oscillatory patterns: folding of limit cycles and existence of chaos, 2) possible scenario of apoptosis, 3) impact of $k_{M A M}$ on the calcium levels on limit cycle and 4) existence and properties of steady states depending on $k_{M A M}$.

In our numerical simulations we have used the MATLAB software. We have used the 3-D version of the model equations i.e. equations (3.8)-(3.10) but on some figures we have depicted additionally CaPr. The steady states were found by the use of the MATLAB Optimization Toolbox and the equilibrium curves were plotted by using MatCont (MATLAB continuation toolbox) [43]. We have observed various types of calcium oscillations (simple, bursting and chaotic ones) for different sets of model parameters. Moreover, we have found sets of parameters for which after a transient time of oscillations, the system 
approaches a steady state with a high mitochondrial calcium level. This can be interpreted as an early step in the apoptotic pathway consisting in sequestering large amount of calcium in mitochondria and the subsequent opening of the permeability transition pores (PTPs) (cf. [8], [44], [45], [46]).

Parameter set leading to bursting oscillations is presented in Table 1 and is the reference case. All the results are calculated for these parameter values unless stated otherwise.
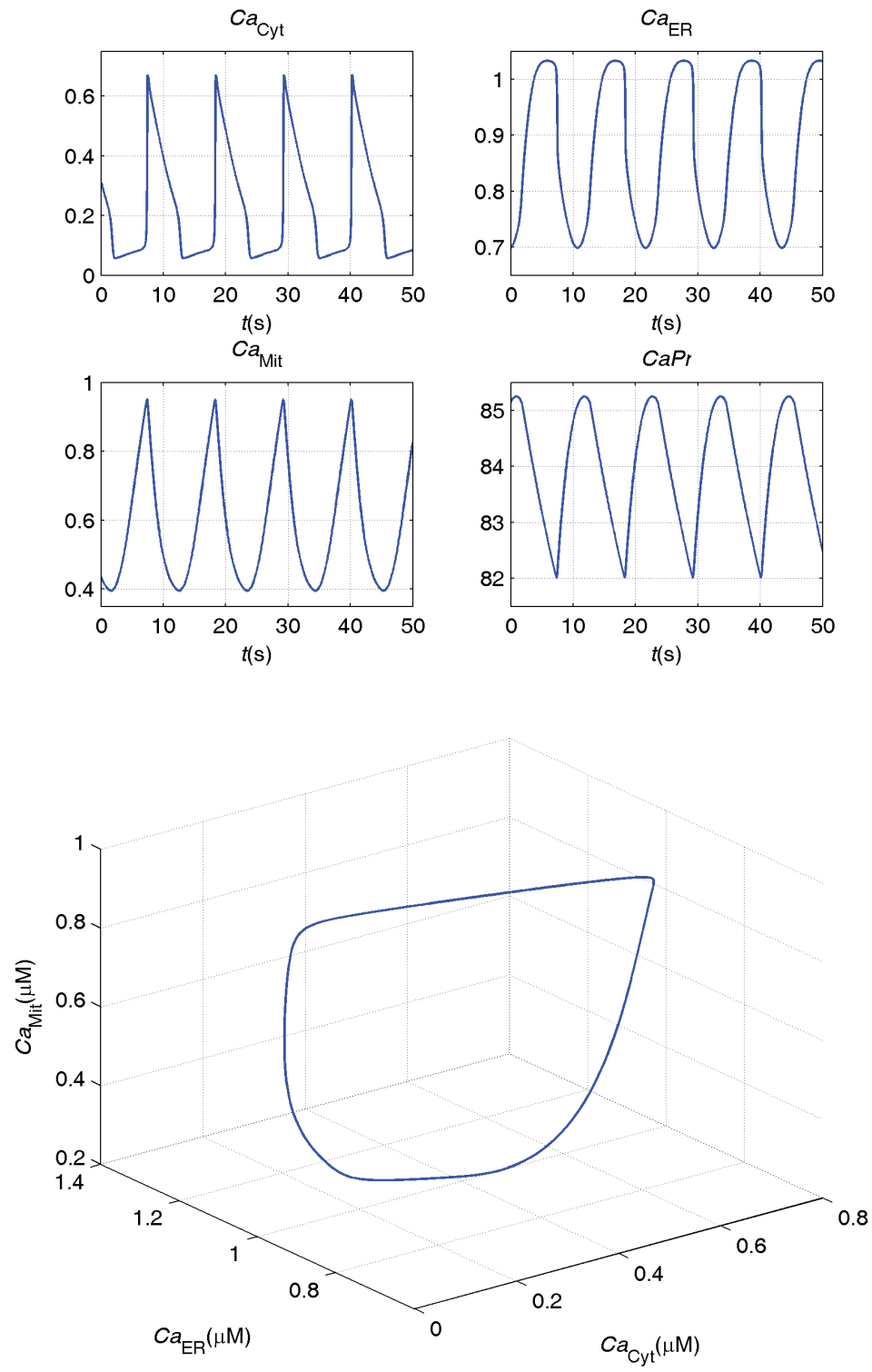

FiguRE 4. (a) - trajectories of calcium concentration changes in specific cell compartments and 3 -D phase portrait for simple oscillations $-K_{2}=1.37, k_{M A M}=1600$ other parameters as in Table 1. 

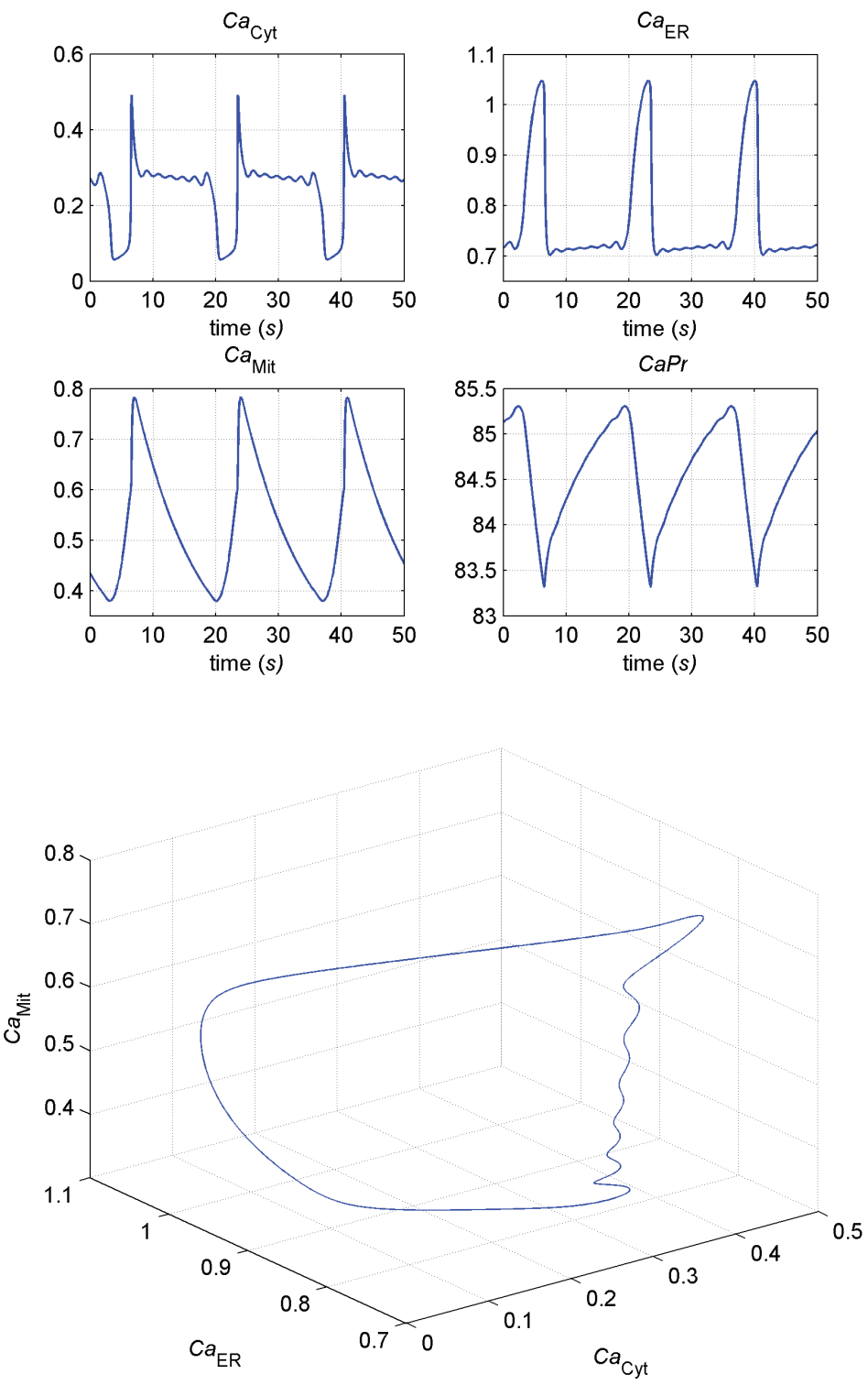

FiguRE 5. (b) - trajectories of calcium concentration changes in specific cell compartments and 3-D phase portrait for bursting oscillations. Parameters as in Table 1

\section{Influence of MAMs on folding of limit cycles and existence of chaos}

The plots of time changes of concentration of $\mathrm{Ca}^{2+}$ ions in specific compartments are given in Figs. 4 and 5. In Fig. 4 we present the time course of the simple oscillations for $K_{2}=1.37$ and $k_{M A M}=1600$, whereas in Fig. 5 bursting calcium oscillations for the parameter set given in Table 1. Let us notice the difference of the period of the limit cycle $T$ (in seconds) and the calcium levels in the specific cellular compartments (in $\mu \mathrm{M}$ ) between the two cases. Thus in Fig. 4: $T=10.9, \mathrm{Ca}_{C y t} \in(0.056,0.56), \mathrm{Ca}_{E R} \in$ $(0.7,1.03), \mathrm{Ca}_{M i t} \in(0.4,0.95)$ and $\mathrm{CaPr} \in(82,85.25)$, whereas in Fig. 5: $T=17.1, \mathrm{Ca}_{C y t} \in(0.057,0.49)$, $\mathrm{Ca}_{E R} \in(0.7,1.05), \mathrm{Ca}_{M i t} \in(0.38,0.78)$ and $\mathrm{CaPr} \in(83.3,85.3)$. Detailed analysis of influence of MAMs on calcium levels in cytosol and mitochondria is presented later in this section. 
Figs. 4 and 5 (lower panels) present also the 3D-phase portraits of the limit cycle for the same sets of parameters as in upper panels of Figs. 4 and 5. In the case of simple oscillations (shown in Fig. 4) there is no high frequency component. The shape of the limit cycles looks similar except for the helix-like part of the trajectory corresponding to the second phase of the single event of bursting calcium oscillations (described below). Fig. 5, which shows the periodic trajectory for $k_{M A M}=1200$, gives an insight into the nature of bursting oscillations. The high frequency, low amplitude part of the trajectory, which resembles a helix (see also insert in Fig. 13), is the consequence of a slow calcium efflux from the mitochondria and the fast exchange of calcium between cytosol, cytosolic buffer proteins and ER.

One period of bursting calcium oscillations is presented in top panel of Fig. 6. The cycle can be divided into three phases. Phase I begins when the level of cytosolic calcium $\left(\mathrm{Ca}_{C y t}\right)$ reaches its maximal value $(t=7.6)$ and continues until the calcium level in mitochondria $\left(\mathrm{Ca}_{M i t}\right)$ reaches its maximal value $(t=8.1)$. In this phase the leading processes are the release of $\mathrm{Ca}^{2+}$ ions from ER and the uptake of calcium ions by mitochondria and cytosolic buffer proteins.

In Phase II, the slow flux of calcium from mitochondria to cytosol takes place. The majority of released calcium is bound by cytosolic buffer proteins. The second process during this phase is the fast calcium exchange between the cytosol and ER, which results in small amplitude, fast oscillations of calcium levels in these compartments. This phase ends when the level of buffered calcium ( $\mathrm{CaPr}$ ) reaches maximum $(t=20.7)$.

In Phase III, the leading process is the dissociation of calcium-protein complexes. The mitochondria and ER are being loaded, while the calcium level in cytosol first decreases and then increases to reach the maximal value, which is the end of Phase III $(t=24.7)$.

It is seen in Figs. 8 and 7 (left panel) that even very low calcium flow through MAMs interface can regularize the trajectories of the model from chaotic $\left(k_{c h}=2950, k_{M A M}=0\right.$ see Fig. 8) to simple ones $\left(k_{c h}=2950, k_{M A M}=3\right.$ see Fig. 7 left panel). In Fig. 8 we present the chaotic trajectory shown in Fig. 5A in [10]. As time increases the trajectories in Figs. 8 and 7 (left panel) are directed clockwise.

However, the influence of the calcium flow through MAMs is far more complex than simply regularizing the trajectories. The behaviour of solutions strongly depends on the value of $k_{M A M}$. We have examined the influence of $k_{M A M}$ on the trajectories for two values of $k_{c h}: 2950$ and 4100 , for which for $k_{M A M}=0$ the asymptotic behaviour of the system is chaotic and simple (stable limit cycle exists), respectively. We have observed stable limit cycles for a wide range of values of $k_{M A M}$; in these cases the largest Lyapunov exponent is 0 and the other two are negative (when the trajectory is chaotic one of the exponents is equal to 0 and at least one is positive). For example, the exponents for $k_{c h}=2950$ and $k_{M A M}=30$ are: $\lambda_{1}=8.9 \times 10^{-6}$ (corresponding to the zero exponent), $\lambda_{2}=-0.07$ and $\lambda_{3}=-4.54$. The time span for the analyzed trajectory, was $5000 \mathrm{~s}$, whereas the time step between Gramm-Schmidt orthonormalizations was chosen $(2 \mathrm{~s})$. We calculated the Lyapunov exponents using MATDS - a MATLAB toolbox for dynamical system investigation (see [47]). However, the numerical computations of Lyapunov exponents are often inaccurate and very sensitive to the choice of the time step of the Gramm-Schmidt orthonormalization. Therefore we often obtained two exponents of different signs and very low magnitude (e.g. of order $10^{-5}$ ), and it was impossible to decide if the trajectory is chaotic or a (folded) limit cycle. In this kind of situations, to distinguish chaotic trajectories, we computed Poincaré section and estimated the correlation dimension $D_{2}$ of the attractor using the Tisean package ([48], [49]). The Poincaré section with respect to the plane $\mathrm{Ca}_{M i t}=1.2$ and the estimated correlation dimension of chaotic trajectory for $k_{c h}=2950, k_{M A M}=73$ is presented in Fig. 9. Since the intersection points of the trajectory with the section plane form a fuzzy quasi arch set (left panel), one may conclude that this trajectory is chaotic [50]. (In the case of periodic solution, this procedure would give one or a couple of points depending on the folding of the limit cycle.) In right panel we have presented the local slopes of the correlation sums for various embedding dimensions $m=1, \ldots, 15$ and box sizes $\varepsilon$ (blue lines). Red line depicts the estimated correlation dimension $D_{2}=1.34$. The estimated correlation dimensions $D_{2}$ (in the denotation used in [48]) for various values ok $k_{M A M}$ parameter and $k_{c h}=2950$ are enlisted in Table 2 . 

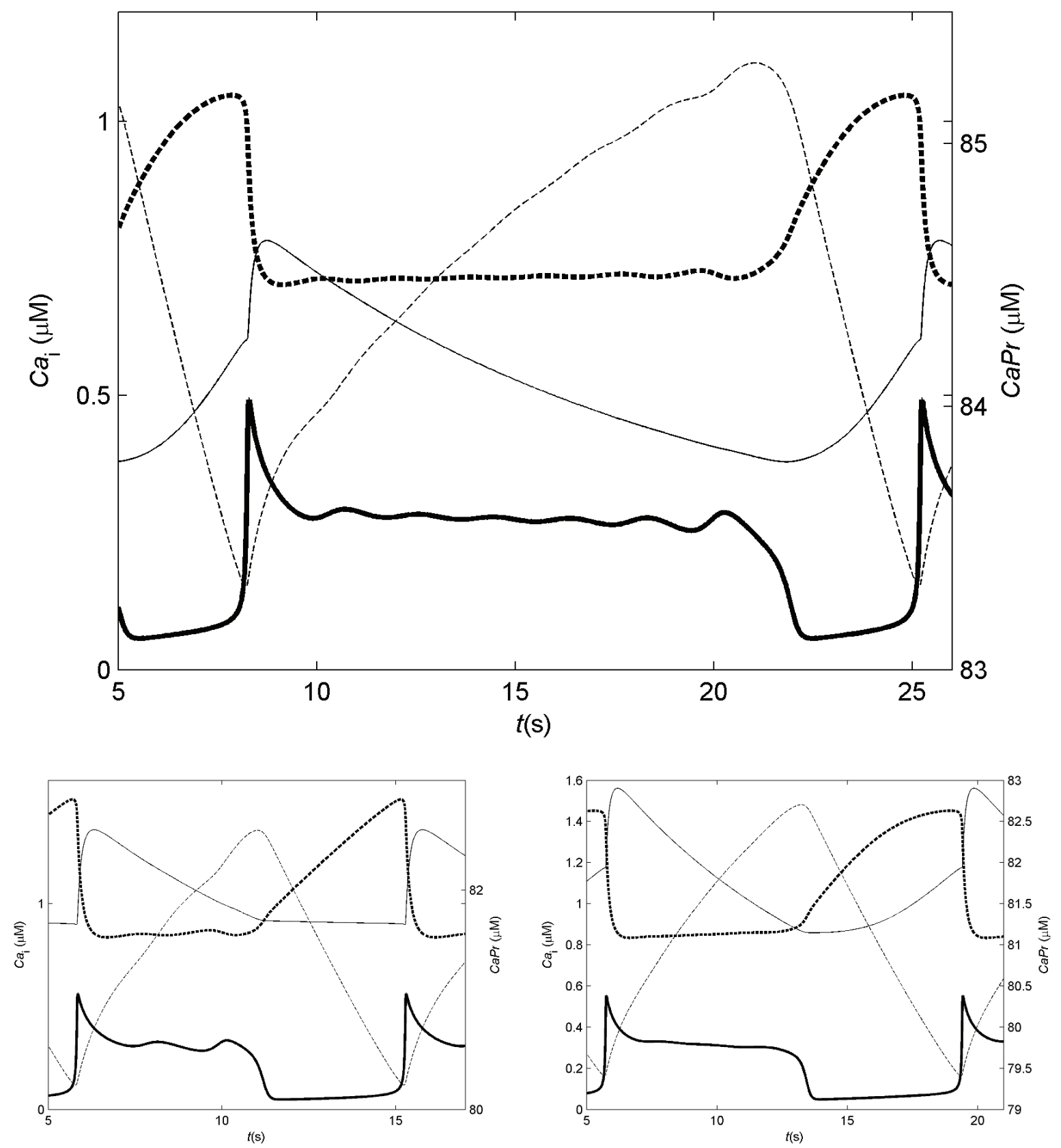

Figure 6 . One period of calcium oscillations: $C a_{C y t}$ - thick, solid line, $C a_{M i t}$ - thin, solid line, $C a_{E R}$ - thick, dashed line, $C a P r$ - thin, dashed line. Binding sites concentration $\mathrm{Pr}$ is related to $\mathrm{CaPR}$ by relation (3.7) and thus not shown here. Top: parameters as in Table 1; bottom left: $k_{c h}=2950, k_{M A M}=3$; bottom right: $k_{c h}=2950, k_{M A M}=87$ 

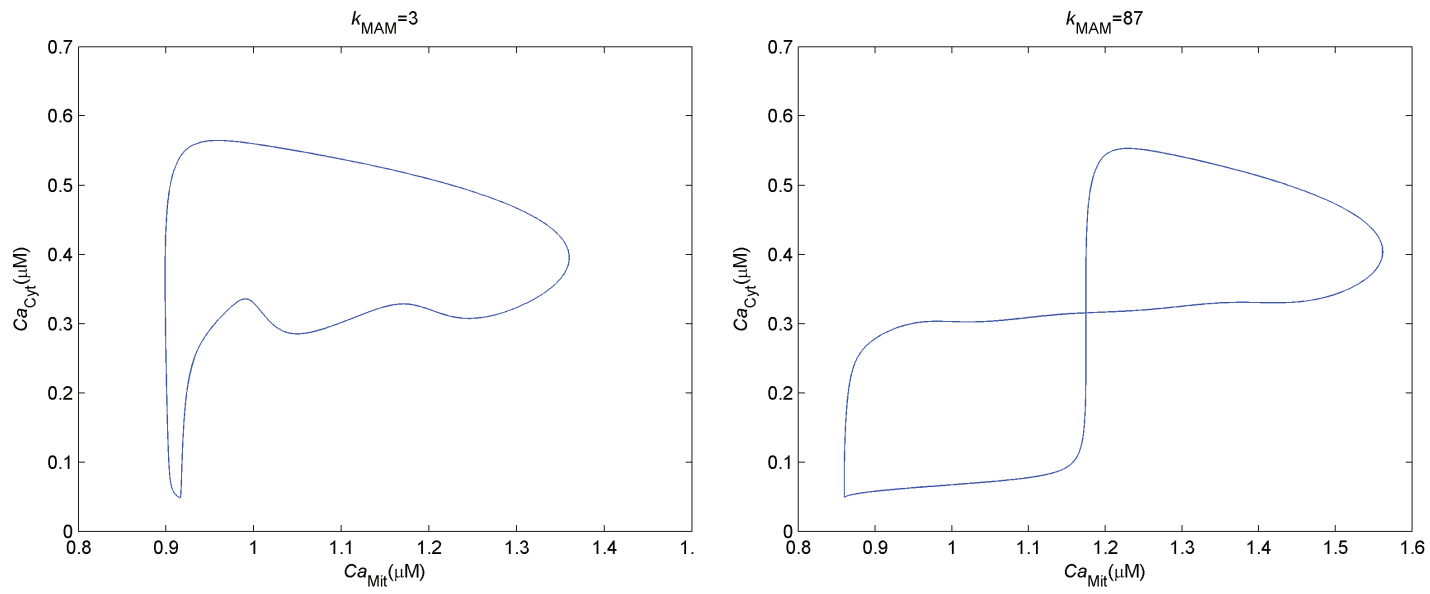

Figure 7. Projections on $\mathrm{Ca}_{M i t}-\mathrm{Ca}_{C y t}$ plane of simple trajectories for $k_{c h}=2950$; left panel $-k_{M A M}=3$, right panel $-k_{M A M}=87$

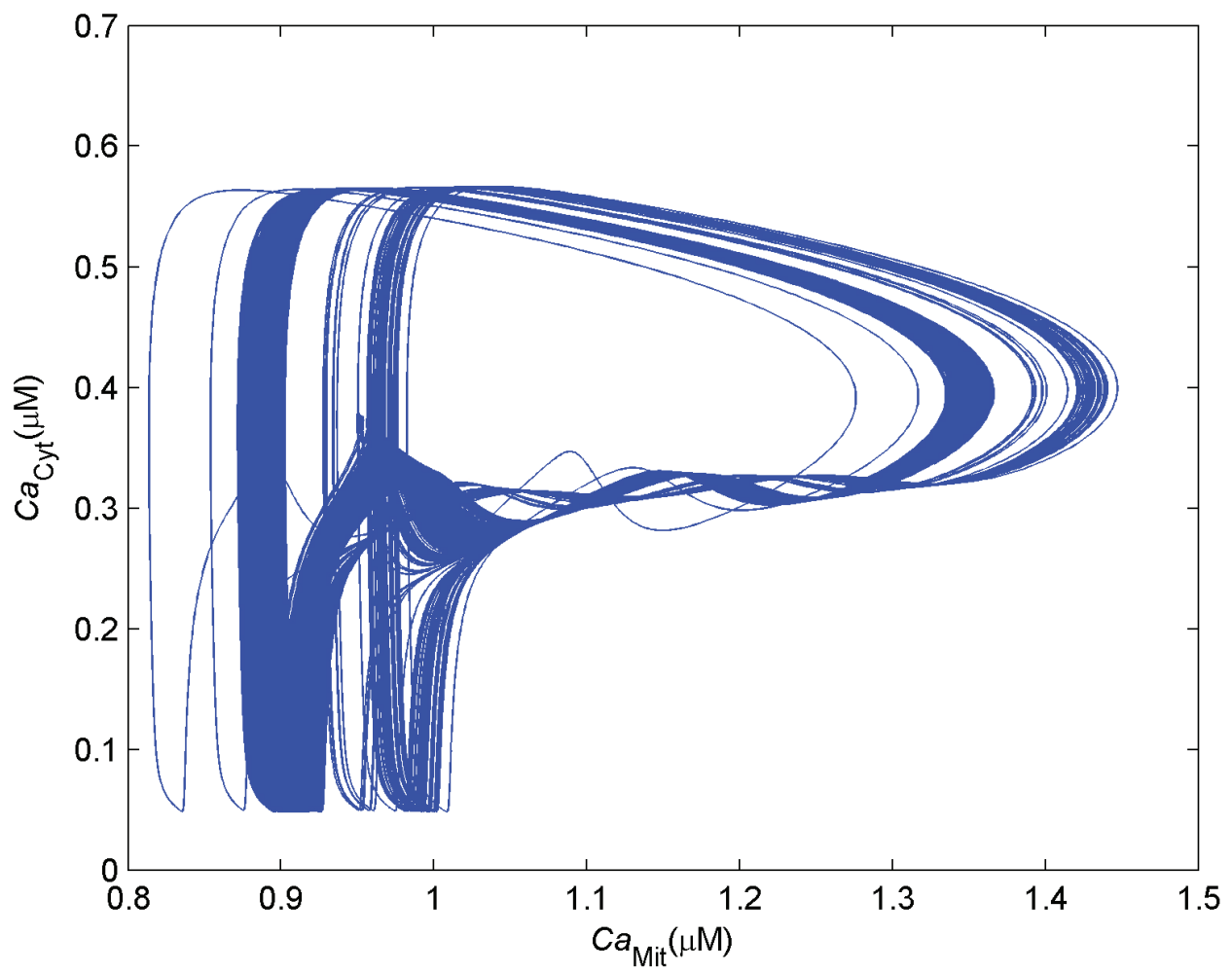

Figure 8. Projection on $\mathrm{Ca}_{M i t}-\mathrm{Ca}_{C y t}$ plane of the chaotic trajectory for $k_{c h}=2950$, $k_{M A M}=0$ (cf. [10])

For $k_{c h}=2950$ we have analyzed the behaviour of solutions for $k_{M A M}$ in the range 0-200 with the step equal to 1 . The value of $k_{M A M}$ influences significantly the type of oscillations. We have observed 1-, 2-, 3- and even 7-fold limit cycles and chaotic oscillations. Moreover, for $k_{M A M} \geq 102$ we did not detect 

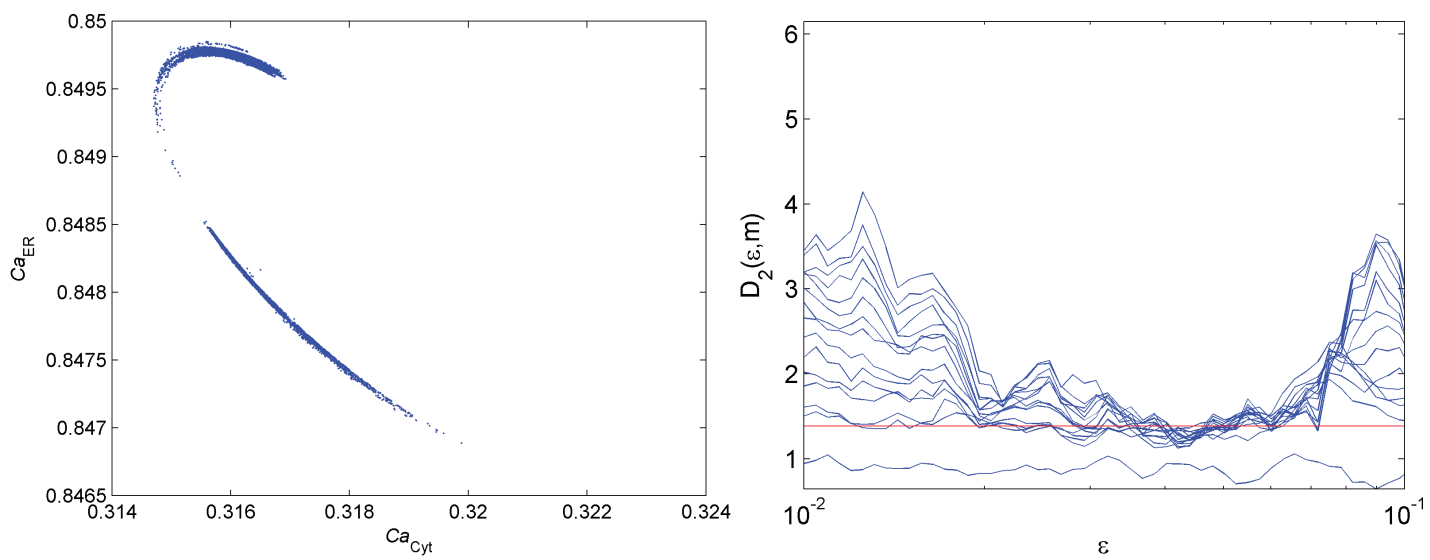

FiguRE 9. Left panel: the Poincaré section with respect to the plane $\mathrm{Ca}_{M i t}=1.2$ for $k_{c h}=2950, k_{M A M}=73$; Right panel: local slopes of correlation sums for different embedding dimensions $m$ (corresponding to each curve) and box sizes $\varepsilon$ - blue lines, estimated correlation dimension $D_{2}=1.34$ - red line (parameters as in the left panel)

\begin{tabular}{|l|c|c|c|c|c|c|c|c|c|}
\hline$k_{M A M}$ & 0 & 41 & 42 & 43 & 46 & 68 & 72 & 73 & 89 \\
\hline$D_{2}$ & 1.84 & 1.02 & 1.05 & 1.12 & 1.64 & 1.1 & 1.02 & 1.34 & 1.09 \\
\hline
\end{tabular}

TABLE 2. Correlation dimensions of attractors for $k_{c h}=2950$.

a stable limit cycle and for those values of $k_{M A M}$ most of the trajectories tend to a stable steady state with overloaded mitochondria $\left(\mathrm{Ca}_{M i t}>18\right)$. This effect has an interesting biological interpretation: it may be regarded as an early phase of an apoptotic pathway (cf. [8], [44], [45]).

The 1-fold limit cycle is observed for $k_{M A M}$ in the ranges 1-39, 74-75 and 86-88. 2-fold limit cycle exists for $k_{M A M}$ in ranges 47-67, 76-85, 90-94, 96-98, 100-101 and for $k_{M A M}$ equal to 40 . The trirythmic calcium oscillations appear for $k_{M A M}$ equal to 69 and 70. Moreover, we have found a 7-fold limit cycle for $k_{M A M}=71$. The evidences of deterministic chaos (either by examining the Lyapunov exponents or the Poincaré section described above) are present for $k_{M A M}$ in ranges $41-46,72-73$ and for $k_{M A M}$ equal to $0,68,89,95,99$ (see Fig. 10 for schematic presentation of this result).

Changes of $k_{M A M}$ can also modify the shape of oscillatory trajectories. For $k_{c h}=2950$ we have considered $k_{M A M}=3$ (illustrated by Fig. 6 bottom left) and $k_{M A M}=87$ (illustrated by Fig. 6 bottom right). In both cases a stable 1 -fold limit cycle exists. For $k_{M A M}=3$ the projection of the trajectory on $\mathrm{Ca}_{M i t}-\mathrm{Ca}_{C y t}$ plane is a single loop as in the left panel of Fig. 7, while for $k_{M A M}=87$ has cipher eight like shape as in the right panel of Fig. 7. Increasing of $k_{M A M}$ causes that the vertical part of the trajectory (the fast cytosol loading at the end of Phase III) moves to higher concentrations of mitochondrial calcium. In these two cases the character of mitochondria loading changes. For $k_{M A M}=3$ we have a fast loading of the mitochondria together with fast loading of the cytosol, while for $k_{M A M}=87$, beside to the fast loading of the mitochondria together with the fast loading of the cytosol there occurs a slow loading of the mitochondria at almost constant, very low calcium level in the cytosol as in Fig. 6. It is interesting that, in this phase, almost the whole of calcium which is sequestered by mitochondria and ER comes from dissociation of calcium-buffer complexes.

Next, we change $k_{M A M}$ from 0 to 1600 with the step equal to 50, keeping the other parameters as in Table 1 (i.e. $k_{c h}=4100$ ). The stable 1 -fold limit cycle exists for $k_{M A M}$ in ranges $0-150,550-850$, 1050-1200, 1300-1400, 1500-1550. The stable 2-fold limit cycle exists for $k_{M A M}$ in ranges 200-500, 9001000 and equal to 1250 and 1450. Chaotic behaviour is observed for $k_{M A M}$ equal to 1600 (see Fig. 10 for schematic presentation of this result). For $k_{M A M} \geq 1649$ the chaotic trajectory loses its stability and 
the majority of trajectories approach the stable steady state with very high mitochondrial calcium level. This effect again can be interpreted as an early phase of the apoptotic pathway.

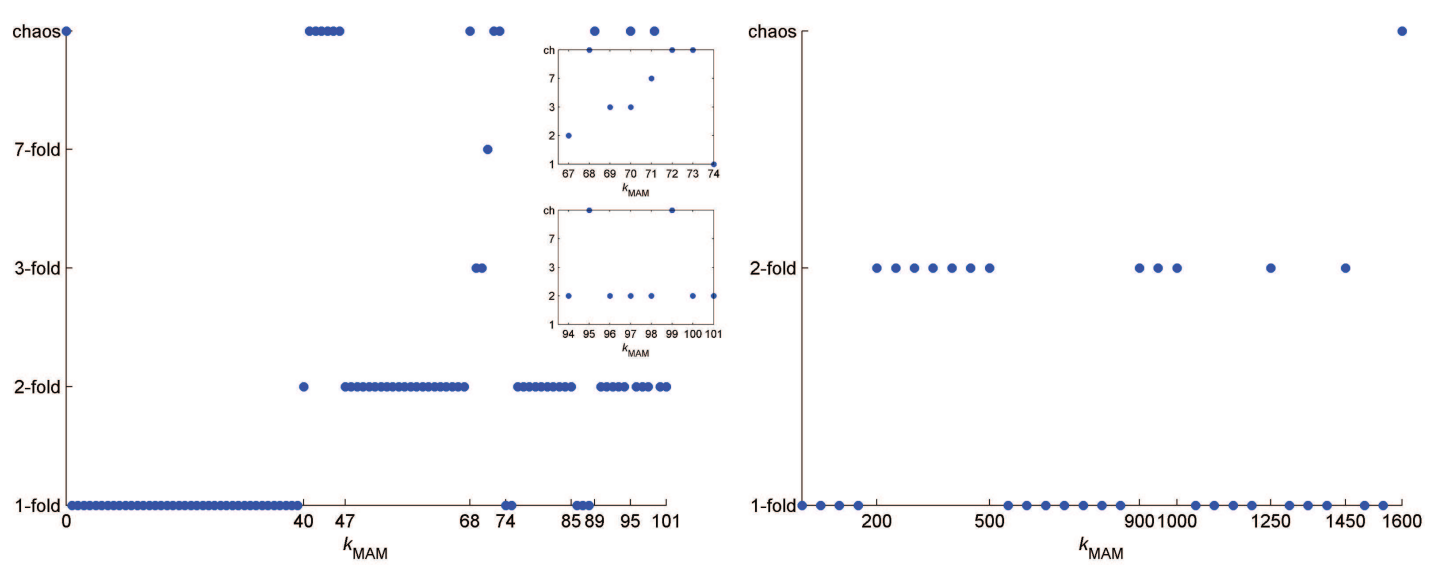

Figure 10. Qualitative picture of non-constant attractors as a function of $k_{M A M}$ for $k_{c h}=2950$ (left) and $k_{c h}=4100$ (right). For different values of $k_{M A M}$ the non-constant attractors may be 1, 2, 3, 7-fold limit cycles or a chaotic trajectory

\section{Biologically acceptable apoptosis scenario}

As mentioned above, we have numerically observed that for $k_{c h}=2950$ and $k_{M A M} \leq 101$ as well as for $k_{c h}=4100$ and $k_{M A M}<1649$ there exist stable limit cycles or chaotic trajectories having large basins of attraction. The qualitative behaviour of the trajectories changes with the increase of $k_{M A M}$. For $k_{c h}=2950$ and $k_{M A M} \geq 102$ as well as for $k_{c h}=4100$ and $k_{M A M} \geq 1649$ the majority of the trajectories tend to the stable steady state with very high mitochondrial calcium level $\mathrm{Ca}_{M i t}$ - point $P_{1}$ in Figs. 12 and 13. One can hypothesize that the increase of $k_{M A M}$ (which defines the strength of calcium flux from ER to mirochondria) may be related to the cellular stress. Experimental data suggest that numerous stress signals are able to increase the amount of MAMs by increasing expression of proteins responsible for ERmitochondria interface formation. For example, Park and collaborators showed in [51] that the number of calreticulin transcripts increases under heat stress condition. Several other reticular chaperones forming MAMs, e.g. erp44, hsp90, grp94, grp75 or sigma-1 receptor are upregulated by starvation, oxidative, heat or ligand induced stress [52], [53], [54]. It is also known that exposure to an acute heat impulse leads to increased glycosylation of CRT [55]. Glycosylation promotes formation of more stable complexes of chaperones, including those building MAMs [56]. The ER chaperone machinery at MAMs is critical for proper handling $\mathrm{Ca}^{2+}$ signals and controls calcium flux into mitochondria. Thus, under chronic stress conditions, mitochondria can uptake large amount of $\mathrm{Ca}^{2+}$. Calcium concentration remains elevated for relatively long period of time, which leads to the collapse of the mitochondrial transmembrane potential $\left(\Delta \Psi_{m}\right)$ and opening of PTPs (permeability transition pores). Rupture of mitochondrial membranes releases cytochrome $\mathrm{C}$ to the cytoplasm, which in turn activates caspase 9 . Caspase 9 initiates the last phase of cell apoptosis resulting in the DNA fragmentation and ultimately cell death [8], [38].

Remark We focus here only on early steps of the apoptotic pathway, characterised as "mitochondria swelling" [29], before the membrane destabilisation and PTPs opening. The opening of PTPs is a kind of "point of no-return" in the programmed death of the cell and leads to crucial physical changes inside the cell. Their modelling would require a much more complicated model than the presented one. Thus, the calcium efflux through PTPs has been omitted in this paper.

\section{Impact of $k_{M A M}$ on the calcium levels}


The plots of minimal and maximal values of $\mathrm{Ca}_{C y t}$ and $\mathrm{Ca}_{M i t}$ in the oscillatory regimes are presented in Fig. 11. The minimal and maximal values of $\mathrm{Ca}_{C y t}$ on the limit cycle remain almost constant for all values of $k_{M A M} \leq 101$ and $k_{M A M} \leq 1600$ for $k_{c h}$ equal to 2950 and 4100 , respectively. On the other hand, for $k_{c h}=2950$ the maximal value of $\mathrm{Ca}_{M i t}$ is generally increasing (up to some perturbations caused by the changes of the oscillatory pattern) while the minimal value of $\mathrm{Ca}_{\mathrm{Mit}}$ does not change monotonically but generally is decreasing. Thus the amplitude of $\mathrm{Ca}_{M i t}$ generally increases with $k_{M A M}$ parameter. For $k_{c h}=4100$ the maximal value of $\mathrm{Ca}_{\text {Mit }}$ generally increases with $k_{M A M}$ and is almost monotonic, while the minimal value remains more or less constant. Thus the amplitude of $\mathrm{Ca}_{M i t}$ is generally increasing with $k_{M A M}$.
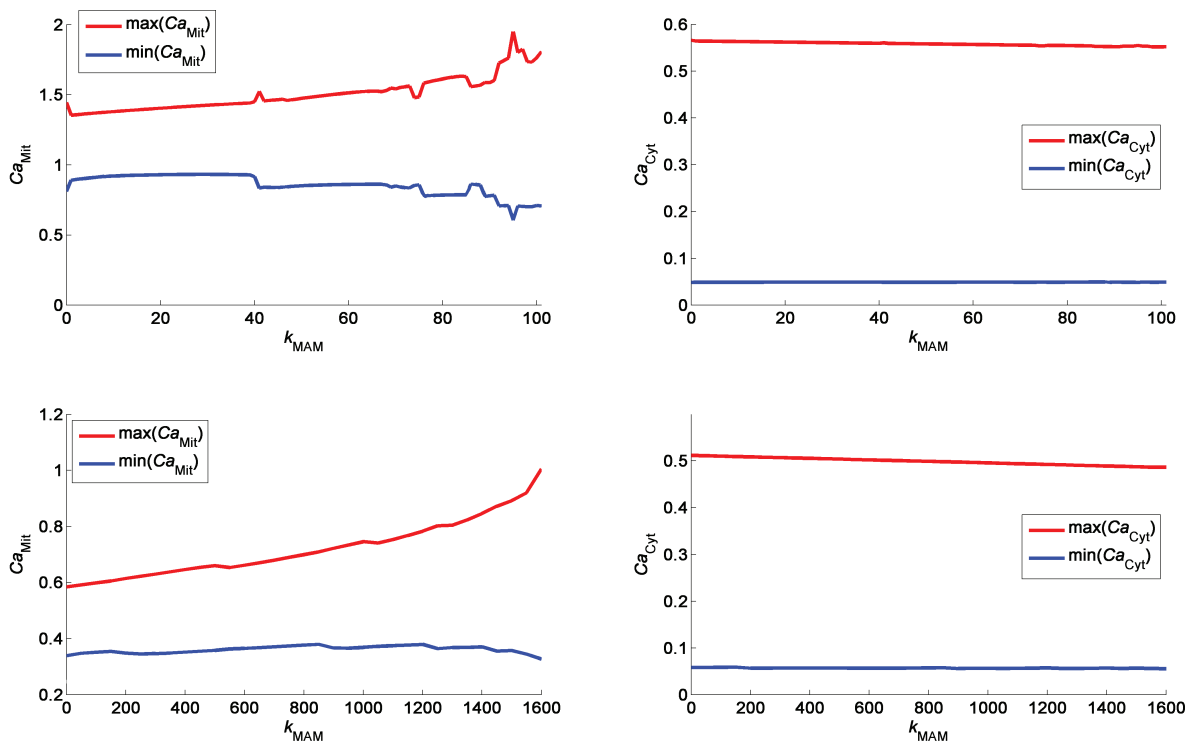

Figure 11. Minimal and maximal values of $\mathrm{Ca}_{M i t}$ (left) and $\mathrm{Ca}_{C y t}$ (right) for oscillatory trajectory. Top row $-k_{c h}=2950$, bottom row $-k_{c h}=4100$

\section{Steady states}

In Fig. 12 we show the dependence of steady states on the parameter $k_{M A M}$ in range 0-10000 (with other parameters as in Table 1). We have found numerically that for $k_{M A M}=0$ (i.e. for the Marhl model) there exists only one non-negative steady state $P_{3 M}=(0.257,0.730,0.102,86.41)$ (blue one), which is unstable. As $k_{M A M}$ increases the saddle-node bifurcation takes place for $k_{M A M} \approx 0.5$ and two new steady states appear: stable $P_{1}$ (green) and unstable $P_{2}$ (red). It is interesting that while the coordinates of $P_{1}$ and $P_{2}$ change significantly with $k_{M A M}$, the coordinates of $P_{3}$ remain almost constant. For example, for $k_{M A M}=1200$, there are the following steady states:

$$
\begin{aligned}
& P_{1}=\left(9.18 \times 10^{-3}, 0.892,19.1,10.1\right) \quad \text { (stable) } \\
& P_{2}=(0.107,1.02,5.94,62.0) \quad \text { (unstable) } \\
& P_{3}=(0.254,0.730,0.173,86.1) \quad \text { (unstable) }
\end{aligned}
$$

In Fig. 13, the steady states $P_{1}, P_{2}$ and $P_{3}$ and some trajectories from their neighbourhood together with the stable limit cycle are shown. As we can see, there exists a separatrix linking $P_{1}$ and $P_{2}$. 


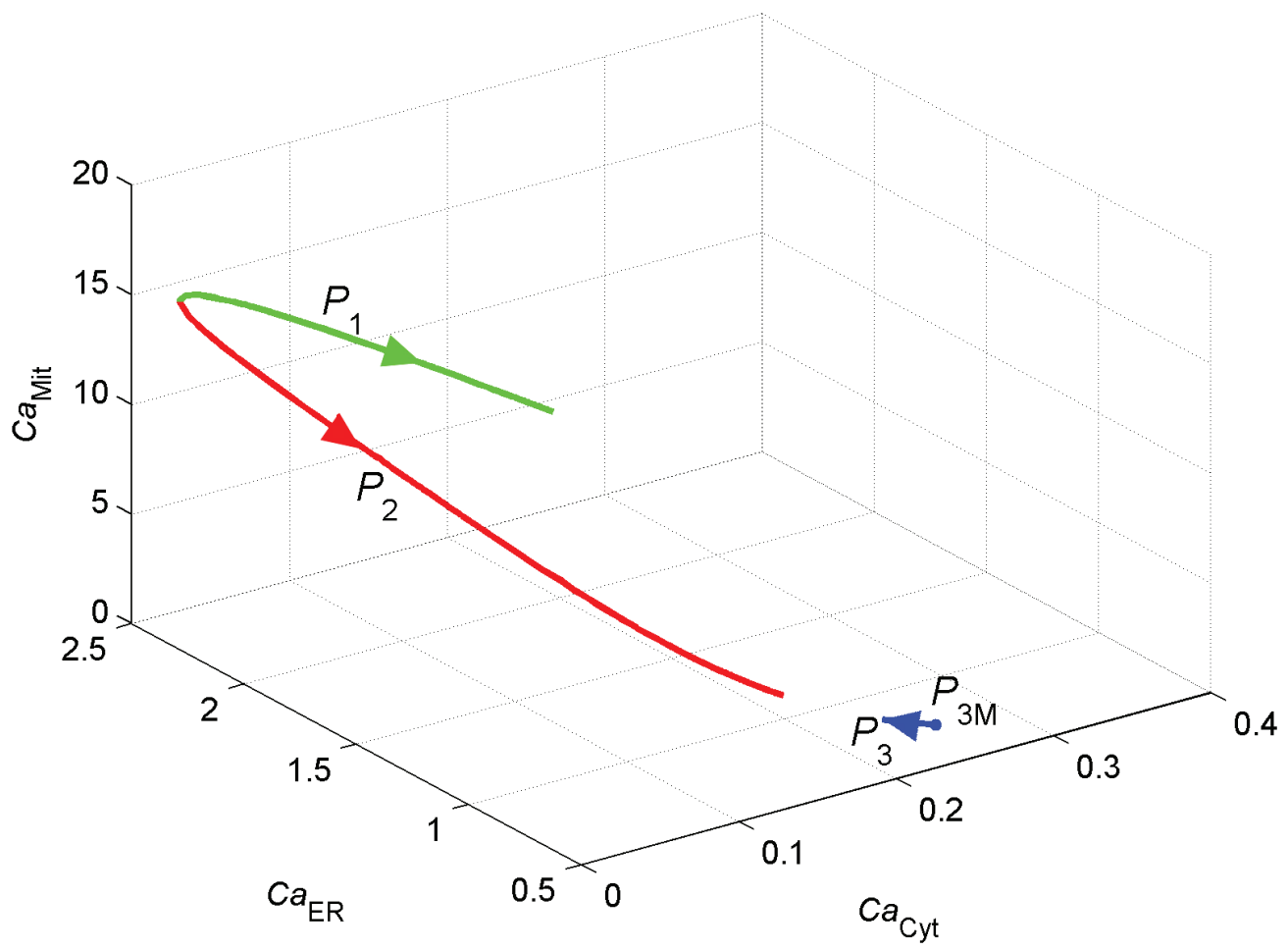

Figure 12. The behaviour of steady states with respect to $k_{M A M} \in[0,10000]: P_{1}-$ green (stable), $P_{2}$ - red (unstable) and $P_{3}$ - blue (unstable). $P_{1}$ and $P_{2}$ are created in a saddle-node bifurcation at $k_{M A M}=0.5$. The arrows indicate the increasing values of $k_{M A M}$

For a wide range of values of $k_{M A M}$ parameter i.e. $k_{M A M} \in(0.5,1649]$ the system exhibits bistable behaviour, that is the two attractors: the stable steady state $P_{1}$ and stable limit cycle/chaotic trajectory co-exist. The coexistence of a stable steady state and a stable limit cycle with large basin of attraction is important from a biological point of view because calcium oscillations are crucial to keep a cell alive. (The protracted elevated calcium concentration in mitochondria usually directs a cell to calcium induced apoptotic pathway.) In Fig. 14, we present the projections of the basin of attraction of $P_{1}$, for parameters as in Table 1, onto the planes $\mathrm{Ca}_{C y t}-\mathrm{Ca}_{E R}, \mathrm{Ca}_{C y t}-\mathrm{Ca}_{M i t}$ and $\mathrm{Ca}_{E R}-\mathrm{Ca} a_{M i t}$. The majority of trajectories of the system is attracted by the stable limit cycle (which means that the oscillations are robust to perturbations), however for large $k_{M A M}$ values (i.e. $\left.k_{M A M} \geq 1650\right) P_{1}$ attracts the vast majority of trajectories. This can be biologically interpreted as mitochondrial calcium accumulation also known as mitochondrial swelling.

\section{Conclusions}

Starting from the model introduced in [10], we have proposed a model of intracellular calcium oscillations, incorporating the existence of mitochondria associated ER membrane complexes (MAMs), the connection sites between endoplasmic reticulum and mitochondria, as a direct flow of calcium ions from ER to mitochondria. We have examined numerically the influence of MAMs on the type and shape of calcium oscillations. We have obtained various oscillatory patterns from simple, through bursting, to chaotic ones. 


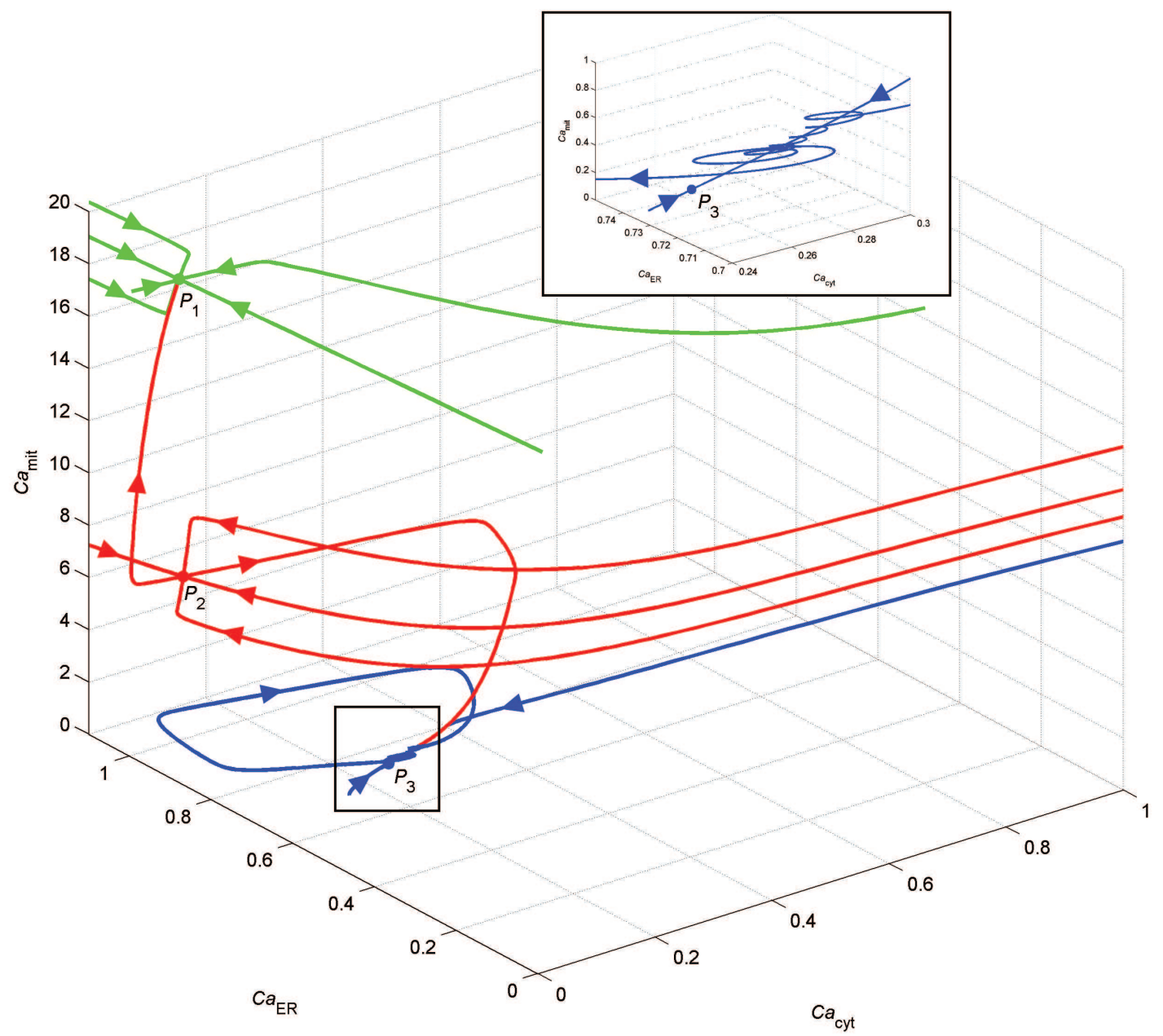

Figure 13. Steady states $P_{1}, P_{2}, P_{3}$ of system (3.8) - (3.10) and the stable limit cycle (parameters as in Table 1). The point $P_{3}$ is hidden under the limit cycle. The insert shows an enlarged neighbourhood of $P_{3}$ with a helix-like part of the limit cycle and stable manifold

Changes of maximal MAMs permeability $\left(k_{M A M}\right)$ lead to changes in the oscillatory pattern. Thus, by increasing $k_{M A M}$ we can regularize the trajectories from chaotic to periodic ones e.g. for $k_{c h}=2950$ and $k_{M A M}=0$ there exists a chaotic trajectory, whereas even for very small values of $k_{M A M}$ the trajectory starting from the same initial point evolves to a stable limit cycle (cf. Fig. 10). The influence of the direct flow of free calcium ions through MAM-interfaces is not restricted to simply regularizing trajectories. There can exist limit cycles, which can be simple, folded or chaotic oscillating trajectories. Moreover, for sufficiently large $k_{M A M}$ stable oscillations disappear. In this case the vast majority of trajectories approach the stable steady state with a high calcium level in mitochondria $-P_{1}$ (the mitochondrial calcium level of $P_{1}$ can be seen in Fig. 12). This can be interpreted as "mitochondria swelling" and an early step in the apoptotic pathway. We have also observed that the increase of $k_{M A M}$ changes the shape of oscillatory patterns, that is the loading of mitochondria starts earlier for larger $k_{M A M}$. Moreover, the amplitude of oscillations and the mean value of $\mathrm{Ca}_{M i t}$ generally increases with $k_{M A M}$. The proposed, biologically reasonable, potential scenario of early steps in the apoptotic pathway is strictly connected with the MAM complexes. 

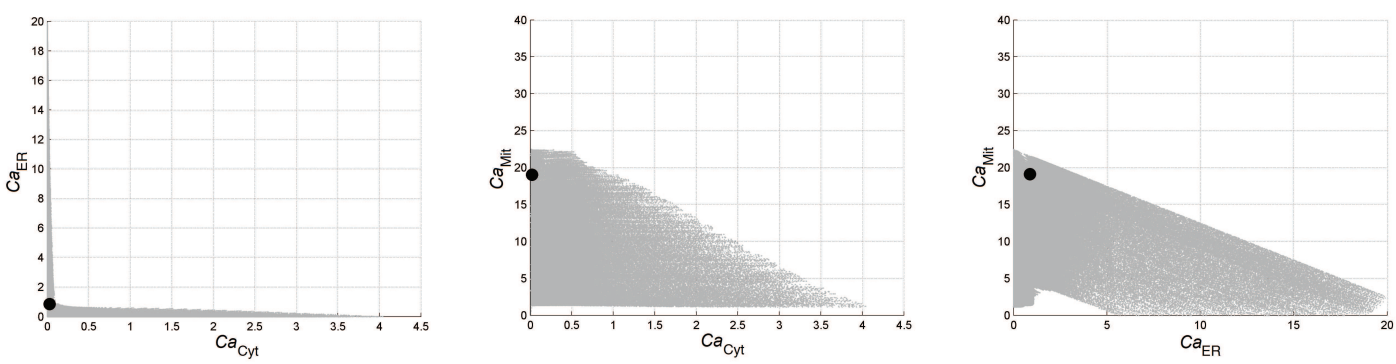

Figure 14. Basin of attraction of $P_{1}$ : projections on planes $\mathrm{Ca}_{C y t}-\mathrm{Ca}_{E R}, \mathrm{Ca}_{C y t}-\mathrm{Ca}_{M i t}$ and $\mathrm{Ca}_{E R}-\mathrm{Ca}_{M i t}$. Black dot corresponds to the stable steady state $P_{1}=(9.18 \times$ $\left.10^{-3}, 0.892,19.1\right)$, gray dots - initial values of trajectories attracted by $P_{1}$. Trajectories starting from the white regions are attracted by the limit cycle

Acknowledgements. This work was supported by FNP project TEAM/2009-3/6 "Mechanistic aspects and spatial organization of cell signaling". B.K. has been also partially supported by the MNiSW grant N N201548738.

\section{References}

[1] D.E. Clapham. Calcium signaling. Cell, 131 (2007), 1047-1058.

[2] A.J. Laude, A.W.M. Simpson. Compartmentalized signalling: Ca2+ compartments, microdomains and the many facets of Ca2+ signalling. FEBS J., 276 (2009), 1800-1816.

[3] C. Montell. The latest waves in calcium signaling. Cell, 122 (2005), 157-163.

[4] A.M. Oster, B. Thomas, D. Terman, C.P Fall. The low conductance mitochondrial permeability transition pore confers excitability and CICR wave propagation in a computational model. J Theor Biol, 273 (2011), 216-231.

[5] G. Hajnóczky, G. Csordás, M. Madesh, P. Pacher. The machinery of local $\mathrm{Ca}^{2+}$ signalling between sarco-endoplasmic reticulum and mitochondria. J. Physiology, 529 (2000), 69-81.

[6] J.E. Chipuk, L. Bouchier-Hayes, D.R. Green. Mitochondrial outer membrane permeabilization during apoptosis: the innocent bystander scenario. Cell Death Differ., 13 (2006) 1396-1400.

[7] S.W. Tait, M.J. Parsons, F. Llambi, L. Bouchier-Hayes, S. Connell, C. Munoz-Pinedo, D.R. Green. Resistance to caspase-independent cell death requires persistence of intact mitochondria. Dev. Cell, 18 (2010) 802-81.

[8] G. Hajnóczky, G. Csordás, S. Das, C. Garcia-Perez, M. Saotome, S.S. Roy, M. Yi. Mitochondrial calcium signalling and cell death: approaches for assessing the role of mitochondrial Ca2+ uptake in apoptosis. Cell Calcium, 40 (2006) $553-560$.

[9] J.A.M. Borghans, G. Dupont, A. Goldbeter. Complex intracellular calcium oscillations. A theoretical exploration of possible mechanisms. Biophysical Chemistry, 66 (1997) 25-41.

[10] M. Marhl, T. Haberichter, M. Brumen, R. Heinrich. Complex calcium oscillations and the role of mitochondria and cytosolic proteins. Biosystems, 57 (2000) 75-86.

[11] G. Csordás, P. Várnai, T. Golenár, S. Roy, G. Purkins, T.G. Schneider, T. Balla, G. Hajnóczky. Imaging interorganelle contacts and local calcium dynamics at the ER-mitochondrial interface. Mol Cell, 39 (2010) 121-132.

[12] E.A. Dennis, E.P. Kennedy. Intracellular sites of lipid synthesis and the biogenesis of mitochondria. J Lipid Res, 13 (1972) 263-267.

[13] A.E. Rusinol, Z. Cui, M.H. Chen, J.E. Vance. A unique mitochondria-associated membrane fraction from rat liver has a high capacity for lipid synthesis and contains pre-Golgi secretory proteins including nascent lipoproteins. J Biol Chem, 269 (1994) 27494-27502.

[14] C. Giorgi, D. De Stefani, A. Bononi, R. Rizzuto, P. Pinton. Structural and functional link between the mitochondrial network and the endoplasmic reticulum. Int J Biochem Cell Biol, 41 (2009) 1817-1827.

[15] M. Lebiedzinska, G. Szabadkai, A.W.E. Jones, J. Duszynski, M.R. Wieckowski. Interactions between the endoplasmic reticulum, mitochondria, plasma membrane and other subcellular organelles. Int J Biochem Cell Biol, 41 (2009) 18051816.

[16] M. Giacomello, I. Drago, M. Bortolozzi, M. Scorzeto, A. Gianelle,P. Pizzo, T. Pozzan. Ca2+ hot spots on the mitochondrial surface are generated by Ca2+ mobilization from stores, but not by activation of store-operated Ca2+ channels. Mol Cell., 38(2) (2010) 280-290.

[17] G. Csordás, C. Renken, P. Várnai, L. Walter, D. Weaver, K.F. Buttle, T. Balla, C.A. Mannella, G. Hajnóczky. Structural and functional features and significance of the physical linkage between ER and mitochondria. J Cell Biol, 174 (2006) 915-921.

[18] T. Hayashi, R. Rizzuto, G. Hajnóczky, T.-P. Su. MAM: more than just a housekeeper. Trends Cell Biol, 19 (2009) $81-88$. 
[19] S. Schuster, M. Marhl, T. Höfer. Modelling of simple and complex calcium oscillations. From single-cell responses to intercellular signalling. Eur J Biochem, 269 (2002) 1333-1355.

[20] D. Hariprasad, M. McNulty, J. Shi, P. Tian. Three-pool model of calcium signaling. https://digitalarchive.wm.edu/ bitstream/handle/10288/1179/Hariprasad $\ \% 20$ Daniel $\ \% 202009$.pdf? sequence=1 (2009).

[21] M. Marhl, S. Schuster, M. Brumen. Mitochondria as an important factor in the maintenance of constant amplitudes of cytosolic calcium oscillations. Biophysical Chemistry, 71 (1998) 125-132.

[22] H. Coe, M. Michalak. Calcium binding chaperones of the endoplasmic reticulum. Gen Physiol Biophys, 28 Spec No Focus (2009) F96-F103.

[23] B. Schwaller. Cytosolic Ca2+ buffers. Cold Spring Harb Perspect Biol, 2(11) a004051.

[24] A.B. Parekh. Mitochondrial regulation of intracellular Ca2+ signaling: more than just simple Ca2+ buffers. News Physiol Sci, 18 (2003) 252-256.

[25] J. Keener, J. Sneyd. Mathematical Physiology, Springer, New York, 1998.

[26] J. Sneyd, A. Duffy, P.D. Dale. Traveling Waves in Buffered Systems: Applications to Calcium Waves. SIAM J. Appl. Math., 58 (1998) 1178-1192.

[27] A. Skupin, M. Falcke. From puffs to global Ca2+ signals: how molecular properties shape global signals. Chaos, 19 (2009) 037111

[28] B.W. Hoogenboom, K. Suda, A. Engel, D. Fotiadis. The supramolecular assemblies of voltage-dependent anion channels in the native membrane. J Mol Biol., 370 (2007) 246-255.

[29] V. Shoshan-Barmatz, V. De Pinto, M. Zweckstetter, Z. Raviv, N. Keinan, N. Arbel. VDAC, a multi-functional mitochondrial protein regulating cell life and death. Mol Aspects Med., 31 (2010) 227-285.

[30] G. Dupont, L. Combettes. What can we learn from the irregularity of Ca2+ oscillations?. Chaos, 19 (2009) 037112.

[31] J. Wagner, J. Keizer. Effects of rapid buffers on Ca2+ diffusion and Ca2+ oscillations. Biophys J., 67 (1994) $447-456$.

[32] A.P. Dawson, G.T. Rich, J.W. Loomis-Husselbee. Estimation of the free [Ca2+] gradient across endoplasmic reticulum membranes by a null-point method. Biochem J., 310 (1995) 371-374.

[33] M. Hoth, C.M. Fanger, R.S. Lewis. Mitochondrial regulation of store-operated calcium signaling in T lymphocytes. J Cell Biol., 137 (1997) 633-648.

[34] Y.-X. Li, J. Keizer, S.S. Stojilkovic, J. Rinzel. Calcium excitability of the ER membrane: an explanation for IP3induced Ca2+ oscillations. Am J Physiol Cell Physiol, 269 (1995) C1079-C1092.

[35] J. Sneyd, K. Tsaneva-Atanasova, D. I. Yule, J. L. Thompson, T. J. Shuttleworth. Control of calcium oscillations by membrane fluxes. Proc Natl Acad Sci USA, 101 (2004) 1392-1396.

[36] D.F. Babcock, B. Hille. Mitochondrial oversight of cellular Ca ${ }^{2+}$ signaling. Curr. Opin. Neurobiol., 8 (1998) $398-404$.

[37] M. Falcke. Reading the patterns in living cells - the physics of Ca2+ signaling Advances in Physics, 53 (2004) $255-440$.

[38] A. Rasola, P. Bernardi, The mitochondrial permeability transition pore and its involvement in cell death and in disease pathogenesis. Apoptosis, 12 (2007) 815-833.

[39] D.F. Babcock, J. Herrington, P.C. Goodwin, Y.B. Park, B. Hille. Mitochondrial participation in the intracellular Ca ${ }^{2+}$ network. J. Cell Biol, 136 (1997) 833-844.

[40] S. Hehl, A. Golard, B. Hille. Involvement of mitochondria in intracellular calcium sequestration by rat gonadotropes. Cell Calcium, 20 (1996) 515-524.

[41] N. Svichar, V. Shishkin, P. Kostyuk. Mitochondrial participation in modulation of calcium transients in DRG neurons. Neuroreport, 10 (1999) 1257-1261.

[42] V.V. Chepyzhov, M.I. Vishik. Attractors for Equations of Mathematical Physics. American Mathematical Society, Providence RI, 2002

[43] W. Govaerts, Yu.A. Kuznetsov, http://www.matcont.ugent.be.

[44] S.K. Joseph, G. Hajnczky. $I P_{3}$ receptors in cell survival and apoptosis: Ca2+ release and beyond. Apoptosis, 12 (2007) 951-968.

[45] S.S. Roy, G. Hajnóczky. Calcium, mitochondria and apoptosis studied by fluorescence measurements. Methods, 46 (2008) 213-223.

[46] R. Rizzuto, P. Pinton, D. Ferrari, M. Chami, G. Szabadkai, P.J. Magalhães, F. Di Virgilio, T. Pozzan. Calcium and apoptosis: facts and hypotheses. Oncogene, 22 (2003) 8619-8627.

[47] V.N. Govorukhin. http://www.math.rsu.ru/mexmat/kvm/matds/.

[48] R. Hegger, H. Kantz, T. Schreiber. http://www.mpipks-dresden.mpg.de/ tisean/Tisean_3.0.1/index.html.

[49] H. Kantz, T. Schreiber. Nonlinear Time Series Analysis, Cambridge University Press, Cambridge, 2004.

[50] A.B. Özer, E. Akin. Tools for detecting chaos. SAU Fen Bilimleri Enstitusu Dergisi, 9 (2005) 60-66.

[51] B.J. Park, D.G. Lee, J.R. Yu, S.K. Jung, K. Choi, J. Lee, J. Lee, Y.S. Kim, J.I. Lee, J.Y. Kwon, J. Lee, A. Singson, W.K. Song, S.H. Eom, C.S. Park, D.H. Kim, J. Bandyopadhyay, J. Ahnn. Calreticulin, a calcium-binding molecular chaperone, is required for stress response and fertility in Caenorhabditis elegans. Mol Biol Cell., 12(9) (2001) 2835-2845.

[52] L. Ellgaard, A. Helenius. ER quality control: towards an understanding at the molecular level. Current Opinion in Cell Biology, 13(4) (2001) 431-437.

[53] T. Anelli, M. Alessio, A. Mezghrani, T. Simmen, F. Talamo, A. Bachi, R. Sitia. ERp44, a novel endoplasmic reticulum folding assistant of thethioredoxin family. The EMBO Journal, 21 (2002) 835-844.

[54] T. Hayashi, T.P. Su. Sigma-1 receptor chaperones at the ER-mitochondrion interface regulate Ca(2+) signaling and cell survival. Cell, 131(3) (2007) 596-610.

[55] S.M. Jethmalani, K.J. Henle. Calreticulin associates with stress proteins: implications for chaperone function during heat stress. J Cell Biochem., 69(1) (1998) 30-43.

[56] L.A. Mizzen, A.N. Kabiling, W.J. Welch. The two mammalian mitochondrial stress proteins, grp 75 and hsp 58, transiently interact with newly synthesized mitochondrial proteins. Cell Regul., 2(2) (1991) 165-179. 